\title{
On continuation criteria for the full compressible Navier-Stokes equations in Lorentz spaces
}

\author{
Yanqing Wang, Wei $\mathrm{Wei}^{\dagger}$, Gang $\mathrm{Wu}^{\ddagger}$ and Yulin $\mathrm{Ye}^{\S}$
}

\begin{abstract}
In this paper, we derive several new sufficient conditions of non-breakdown of strong solutions for both the 3D heat-conducting compressible Navier-Stokes system and nonhomogeneous incompressible Navier-Stokes equations. First, it is shown that there exists a positive constant $\varepsilon$ such that the solution $(\rho, u, \theta)$ to full compressible NavierStokes equations can be extended beyond $t=T$ provided that one of the following two conditions holds

(1) $\rho \in L^{\infty}\left(0, T ; L^{\infty}\left(\mathbb{R}^{3}\right)\right), u \in L^{p, \infty}\left(0, T ; L^{q, \infty}\left(\mathbb{R}^{3}\right)\right)$ and

$$
\|u\|_{L^{p, \infty}\left(0, T ; L^{q, \infty}\left(\mathbb{R}^{3}\right)\right)} \leq \varepsilon, \text { with } 2 / p+3 / q=1, \quad q>3 ;
$$

(2) $\lambda<3 \mu, \rho \in L^{\infty}\left(0, T ; L^{\infty}\left(\mathbb{R}^{3}\right)\right), \theta \in L^{p, \infty}\left(0, T ; L^{q, \infty}\left(\mathbb{R}^{3}\right)\right)$ and

$$
\|\theta\|_{L^{p, \infty}\left(0, T ; L^{q, \infty}\left(\mathbb{R}^{3}\right)\right)} \leq \varepsilon, \text { with } 2 / p+3 / q=2, \quad q>3 / 2 .
$$

To the best of our knowledge, this is the first continuation theorem allowing the time direction to be in Lorentz spaces for the compressible fluid. Second, we establish some blow-up criteria in anisotropic Lebesgue spaces, for the finite blow-up time $T^{*}$,

(1) Assume that the pair $(p, \vec{q})$ satisfies $2 / p+1 / q_{1}+1 / q_{2}+1 / q_{3}=1\left(1<q_{i}<\infty\right)$ and (1.17), then

$$
\limsup _{t \rightarrow T^{*}}\left(\|\rho\|_{L^{\infty}\left(0, t ; L^{\infty}\left(\mathbb{R}^{3}\right)\right)}+\|u\|_{L^{p}\left(0, t ; L_{1}^{q_{1}} L_{2}^{q_{2}} L_{3}^{q_{3}}\left(\mathbb{R}^{3}\right)\right)}\right)=\infty,
$$

(2) Let the pair $(p, \vec{q})$ satisfy $2 / p+1 / q_{1}+1 / q_{2}+1 / q_{3}=2\left(1<q_{i}<\infty\right)$ and (1.17), then

$$
\limsup _{t \rightarrow T^{*}}\left(\|\rho\|_{L^{\infty}\left(0, t ; L^{\infty}\left(\mathbb{R}^{3}\right)\right)}+\|\theta\|_{L^{p}\left(0, t ; L_{1}^{q_{1}} L_{2}^{q_{2}} L_{3}^{q_{3}}\left(\mathbb{R}^{3}\right)\right)}\right)=\infty,(\lambda<3 \mu) .
$$

Third, without the condition on $\rho$ in (0.1) and (0.3), the results also hold for the 3D nonhomogeneous incompressible Navier-Stokes equations. The appearance of vacuum in these systems could be allowed.
\end{abstract}

MSC(2000): 76D03, 76D05, 35B33, 35Q35

Keywords: Navier-Stokes equations; weak solutions; regularity

\footnotetext{
${ }^{*}$ Department of Mathematics and Information Science, Zhengzhou University of Light Industry, Zhengzhou, Henan 450002, P. R. China Email: wangyanqing20056@gmail.com

${ }^{\dagger}$ Center for Nonlinear Studies, School of Mathematics, Northwest University, Xi'an, Shaanxi 710127, P. R. China Email: ww5998198@126.com

${ }^{\ddagger}$ School of Mathematical Sciences, University of Chinese Academy of Sciences, Beijing 100049, P. R. China Email: wugang2011@ucas.ac.cn

${ }^{\S}$ School of Mathematics and Statistics, Henan University, Kaifeng, 475004, P. R. China Email: ylye@vip.henu.edu.cn
} 


\section{Introduction}

In this paper, we are concerned with the following 3D full compressible Navier-Stokes equations

$$
\left\{\begin{array}{l}
\rho_{t}+\nabla \cdot(\rho u)=0 \\
\rho u_{t}+\rho u \cdot \nabla u+\nabla P(\rho, \theta)-\mu \Delta u-(\mu+\lambda) \nabla \operatorname{div} u=0 \\
c_{v}\left[\rho \theta_{t}+\rho u \cdot \nabla \theta\right]+P \operatorname{div} u-\kappa \Delta \theta=\frac{\mu}{2}\left|\nabla u+(\nabla u)^{\operatorname{tr}}\right|^{2}+\lambda(\operatorname{div} u)^{2}, \\
\left.(\rho, u, \theta)\right|_{t=0}=\left(\rho_{0}, u_{0}, \theta_{0}\right),
\end{array}\right.
$$

where $\rho, u, \theta$ stand for the flow density, velocity and the absolute temperature, respectively. The scalar function $P$ represents the pressure, the state equation of which is determined by

$$
P=R \rho \theta, R>0,
$$

and $\kappa$ is a positive constant. $\mu$ and $\lambda$ are the coefficients of viscosity, which are assumed to be constants, satisfying the following physical restrictions:

$$
\mu>0,2 \mu+3 \lambda \geq 0
$$

The initial conditions satisfy

$$
\rho(x, t) \rightarrow 0, u(x, t) \rightarrow 0, \theta(x, t) \rightarrow 0, \text { as }|x| \rightarrow \infty, \text { for } t \geq 0 .
$$

It is clear that if the triplet $(\rho(x, t), u(x, t), \theta(x, t))$ solve system (1.1), then the triplet $\left(\rho_{\lambda}, u_{\lambda}, \theta_{\lambda}\right)$ is also a solution of (1.1) for any $\lambda \in \mathbb{R}^{+}$, where

$$
\rho_{\lambda}=\rho\left(\lambda x, \lambda^{2} t\right), \quad u_{\lambda}=\lambda u\left(\lambda x, \lambda^{2} t\right), \quad \theta_{\lambda}=\lambda^{2} \theta\left(\lambda x, \lambda^{2} t\right) .
$$

The scaling of velocity $u$ is the same as the incompressible Navier-Stokes system

$$
\left\{\begin{array}{l}
u_{t}-\Delta u+u \cdot \nabla u+\nabla \Pi=0 \\
\operatorname{div} u=0 \\
\left.u\right|_{t=0}=u_{0}
\end{array}\right.
$$

In contrast with the pressure $P=R \rho \theta$ in (1.1), the pressure $\Pi$ in (1.6) is determined by $\Delta \Pi=-\operatorname{div} \operatorname{div}(u \otimes u)$. The global well-posedness of the 3D Navier-Stokes equations (1.6) is an outstanding problem. It is well known that the solution $u$ to (1.6) is regular on $(0, T]$ if $u$ satisfy

$$
u \in L^{p}\left(0, T ; L^{q}\left(\mathbb{R}^{3}\right)\right) \quad \text { with } \quad 2 / p+3 / q=1, \quad q>3 .
$$

This is so-called Serrin type regularity criteria for the incompressible Navier-Stokes system obtained in [27, 31]. Since Lorentz spaces $L^{r, s}\left(\mathbb{R}^{3}\right)(s \geq r)$ are larger than the Lebesgue spaces $L^{r}\left(\mathbb{R}^{3}\right)$ and enjoy the same scaling as $L^{r}\left(\mathbb{R}^{3}\right)$, Chen-Price [5], Sohr [32], and KozonoKim [20] presented an improvement of (1.7) by

$$
u \in L^{p, \infty}\left(0, T ; L^{q, \infty}\left(\mathbb{R}^{3}\right)\right) \text { and }\|u\|_{L^{p, \infty}\left(0, T ; L^{q, \infty}\left(\mathbb{R}^{3}\right)\right)} \leq \varepsilon \text { with } 2 / p+3 / q=1, \quad q>3 .
$$

Alternative proofs of (1.8) are given in [3, 12, 35].

We turn our attention back to the full compressible fluid (1.1). The classical theory of compressible flow can be found in [10, 23]. Here, we are concerned with the regularity of 
local strong solutions to equations (1.1) established by Cho and Kim in 7] (For details, see Proposition 2.3 in Section 2). This type of strong solution allows the initial data to be in vacuum. In the spirit of (1.7), many authors successfully extended Serrin type blowup criteria (1.7) to the compressible flow (e.g. [9, 15, 16, 18, 22, 22, 28, 29, 37, 38] and references therein). In particular, Huang, Li and Wang [15] proved the following necessary condition for blow-up of solutions to (1.1)

$$
\lim \sup _{t \rightarrow T^{\star}}\left(\|\operatorname{div} u\|_{L^{1}\left(0, t ; L^{\infty}\left(\mathbb{R}^{3}\right)\right)}+\|u\|_{L^{p}\left(0, t ; L^{q}\left(\mathbb{R}^{3}\right)\right)}\right)=\infty, \frac{2}{p}+\frac{3}{q}=1, q>3,
$$

where $0<T^{*}<\infty$ is the maximal time of existence of a strong solution of system (1.1). Subsequently, in [14], Huang and Li improved (1.9) to

$$
\lim \sup _{t \rightarrow T^{\star}}\left(\|\rho\|_{L^{\infty}\left(0, t ; L^{\infty}\left(\mathbb{R}^{3}\right)\right)}+\|u\|_{L^{p}\left(0, t ; L^{q}\left(\mathbb{R}^{3}\right)\right)}\right)=\infty, \frac{2}{p}+\frac{3}{q}=1, q>3 .
$$

Very recently, authors in [18] showed that, if $\lambda<3 \mu$, (1.10) can be replaced by

$$
\lim \sup _{t \rightarrow T^{\star}}\left(\|\rho\|_{L^{\infty}\left(0, t ; L^{\infty}\left(\mathbb{R}^{3}\right)\right)}+\|\theta\|_{L^{p}\left(0, t ; L^{q}\left(\mathbb{R}^{3}\right)\right)}\right)=\infty, \frac{2}{p}+\frac{3}{q}=2, q>3 / 2 .
$$

We would like to point out that the norms in (1.9)-(1.11) are scaling-invariant in the sense of (1.5). Inspired by (1.8), we shall prove the following result

Theorem 1.1. Suppose that $(\rho, u, \theta)$ is the unique strong solution to (1.1). Then there exists a positive constant $\varepsilon$ such that the solution $(\rho, u, \theta)$ can be extended beyond $t=T$ provided that one of the following two conditions holds

(1) $\rho \in L^{\infty}\left(0, T ; L^{\infty}\left(\mathbb{R}^{3}\right)\right), u \in L^{p, \infty}\left(0, T ; L^{q, \infty}\left(\mathbb{R}^{3}\right)\right)$ and

$$
\|u\|_{L^{p, \infty}\left(0, T ; L^{q, \infty}\left(\mathbb{R}^{3}\right)\right)} \leq \varepsilon, \quad \text { with } 2 / p+3 / q=1, \quad q>3 ;
$$

(2) $\lambda<3 \mu, \rho \in L^{\infty}\left(0, T ; L^{\infty}\left(\mathbb{R}^{3}\right)\right), \theta \in L^{p, \infty}\left(0, T ; L^{q, \infty}\left(\mathbb{R}^{3}\right)\right)$ and

$$
\|\theta\|_{L^{p, \infty}\left(0, T ; L^{q, \infty}\left(\mathbb{R}^{3}\right)\right)} \leq \varepsilon, \quad \text { with } 2 / p+3 / q=2, \quad q>3 / 2 .
$$

Remark 1.1. Theorem 1.1 generalizes both continuation criteria (1.10) and (1.11) to enable their space-time directions to be in Lorentz spaces.

Remark 1.2. Even if the results in this theorem reduce to the ones only involving space direction in Lorentz spaces, they are still new. Note that the known best blow-up criteria based on Lorentz spaces for the isentropic compressible Navier-Stokes flows were established by $\mathrm{Xu}$ and Zhang [38]

$$
\lim \sup _{t \rightarrow T^{\star}}\left(\|\rho\|_{L^{\infty}\left(0, t ; L^{\infty}\left(\mathbb{R}^{3}\right)\right)}+\|u\|_{L^{p}\left(0, t ; L^{q, \infty}\left(\mathbb{R}^{3}\right)\right)}\right)=\infty, \frac{2}{p}+\frac{3}{q}=1, q>3 .
$$

Hence, it seems that Theorem 1.1 is the first continuation criteria allowing the time direction to be in Lorentz spaces for the compressible fluid.

Remark 1.3. Since $\operatorname{div} u \in L^{1}\left(0, t ; L^{\infty}\left(\mathbb{R}^{3}\right)\right)$ yields $\rho \in L^{\infty}\left(0, t ; L^{\infty}\left(\mathbb{R}^{3}\right)\right), \quad \rho \in$ $L^{\infty}\left(0, t ; L^{\infty}\left(\mathbb{R}^{3}\right)\right)$ in this theorem can be replaced by $\operatorname{div} u \in L^{1}\left(0, t ; L^{\infty}\left(\mathbb{R}^{3}\right)\right)$. Therefore, this theorem is an extention of (1.9). 
The absolute continuity of norm in Lorentz space $L^{p, l}(0, T)(l<\infty)$ together with Theorem 1.1 yields that

Corollary 1.2. Suppose $(\rho, u, \theta)$ is the unique strong solution to (1.1). Then the strong solution can be extended beyond $t=T$ if one of the following two conditions hold: for $l<\infty$,

(1) $\rho \in L^{\infty}\left(0, T ; L^{\infty}\left(\mathbb{R}^{3}\right)\right), u \in L^{p, l}\left(0, T ; L^{q, \infty}\left(\mathbb{R}^{3}\right)\right)$ with $2 / p+3 / q=1, \quad q>3$;

(2) $\lambda<3 \mu, \rho \in L^{\infty}\left(0, T ; L^{\infty}\left(\mathbb{R}^{3}\right)\right), \theta \in L^{p, l}\left(0, T ; L^{q, \infty}\left(\mathbb{R}^{3}\right)\right)$ with $2 / p+3 / q=2, \quad q>3 / 2$.

Owing to the existence of density in (1.1), it seems to be difficult to apply the regularity estimates for heat equations and vorticity equations of (1.6) as used in [20, 32] to show Theorem 1.1. Our strategy is to adopt the method introduced by Bosia, Pata and Robinson in [3] and recently developed in [19]. In this procedure, the key point is to derive an estimate in terms of the following form, for $\phi(t) \geq 0$,

$$
\frac{d}{d t} \phi(t) \leq C\|u\|_{L^{q, \infty}\left(\mathbb{R}^{3}\right)}^{\frac{2 q}{q-3}} \phi(t) .
$$

We observed that, by means of temperature equation, the critical estimate for (1.11) in [18] is that, there exists $\psi(t) \geq 0$ such that

$$
\frac{d}{d t} \psi(t) \leq C\|\theta\|_{L^{q\left(\mathbb{R}^{3}\right)}}^{\frac{2 q}{2 q-3}} \psi(t)
$$

Based on this, invoking the total energy $E=\theta+\frac{|u|^{2}}{2}$ as [14], we can obtain energy estimates in terms of (1.15). Then, Sobolev's inequality in Lorentz spaces and boundedness of Riesz transform in Lorentz spaces allow us to further derive that

$$
\frac{d}{d t} \phi(t) \leq C\|u\|_{L^{q, \infty}\left(\mathbb{R}^{3}\right)}^{\frac{2 q(1-\tau)}{q-3}} \phi^{1+2 \tau}(t) .
$$

Subsequently, we can apply the general Gronwall inequality in Lemma 2.1 and Lemma 2.2 to complete the proof. Finally, we would like to point out that it is unknown whether (1.15) holds for the isentropic compressible Navier-Stokes system.

On the other hand, authors in 34] recently obtained the following local regularity criteria in anisotropic Lebesgue spaces for suitable weak solutions to the incompressible NavierStokes system (1.6)

$$
u \in L_{t}^{p} L_{x}^{\vec{q}}(Q(\varrho)), \quad \text { with } \quad \frac{2}{p}+\sum_{j=1}^{3} \frac{1}{q_{j}}=1, \quad 1<q_{j}<\infty .
$$

This enlightened us to consider the regularity of strong solutions to the compressible NavierStokes equations (1.1) in anisotropic Lebesgue spaces. Before formulating our result, we write

$$
\Xi=\left\{\left(q_{1}, q_{2}, q_{3}\right): \frac{1}{q_{i}}+\frac{1}{q_{j}} \neq \frac{1}{q_{k}}, 1<q_{i}, q_{j}, q_{k}<\infty, i, j, k \in\{1,2,3\}\right\} .
$$

Theorem 1.3. Let $(\rho, u, \theta)$ be the unique strong solution to (1.1) and $\vec{q}=\left(q_{1}, q_{2}, q_{3}\right)$. Then, 
(1)

$$
\limsup _{t \rightarrow T^{*}}\left(\|\rho\|_{L^{\infty}\left(0, t ; L^{\infty}\left(\mathbb{R}^{3}\right)\right)}+\|u\|_{L^{p}\left(0, t ; L^{\vec{q}}\left(\mathbb{R}^{3}\right)\right)}\right)=\infty,
$$

where the pair $(p, \vec{q})$ meets (1.17) and

$$
\frac{2}{p}+\frac{1}{q_{1}}+\frac{1}{q_{2}}+\frac{1}{q_{3}}=1, \quad 1<q_{i}<\infty ;
$$

(2) for $\lambda<3 \mu$,

$$
\limsup _{t \rightarrow T^{*}}\left(\|\rho\|_{L^{\infty}\left(0, t ; L^{\infty}\left(\mathbb{R}^{3}\right)\right)}+\|\theta\|_{L^{p}\left(0, t ; L^{\vec{q}}\left(\mathbb{R}^{3}\right)\right)}\right)=\infty,
$$

where the pair $(p, \vec{q})$ meets (1.17) and

$$
\frac{2}{p}+\frac{1}{q_{1}}+\frac{1}{q_{2}}+\frac{1}{q_{3}}=2, \quad 1<q_{i}<\infty .
$$

Remark 1.4. In contrast with (1.16), (1.18) requires the additional condition (1.17). The reason is due to the application of the boundness of singular integral operator in anisotropic Lebesgue spaces.

Next, we demonstrate that the above proof can be applied to the following nonhomogeneous incompressible Navier-Stokes equations

$$
\left\{\begin{array}{l}
\rho_{t}+\nabla \cdot(\rho u)=0 \\
\rho u_{t}+\rho u \cdot \nabla u-\Delta u+\nabla \Pi=0, \\
\operatorname{div} u=0 \\
\left.u\right|_{t=0}=u_{0},
\end{array}\right.
$$

The local existence of strong solutions to (1.20) in sense of Proposition 2.4 was established by Choe and Kim in [8]. In addition, they showed, if $T^{*}$ is the finite blow-up time, then

$$
\limsup _{t \rightarrow T^{*}}\|\nabla u\|_{L^{4}\left(0, t ; L^{2}\left(\mathbb{R}^{3}\right)\right)}=\infty .
$$

Kim [21] extended Serrin type blow-up criteria to system (1.20) and showed

$$
\limsup _{t \rightarrow T^{*}}\|u\|_{L^{p}\left(0, t ; L^{q, \infty}\left(\mathbb{R}^{3}\right)\right)}=\infty, \quad \text { with } 2 / p+3 / q=2, q>3 .
$$

Our result concerning nonhomogeneous incompressible Navier-Stokes equations (1.20) is

Theorem 1.4. Assume that $(\rho, u)$ is the unique strong solution to (1.20). Then there exists a positive constant $\varepsilon$ such that the solution $(\rho, u)$ can be extended beyond $t=T$ provided that one of the following three conditions holds

(1) $u \in L^{p, \infty}\left(0, T ; L^{q, \infty}\left(\mathbb{R}^{3}\right)\right)$ and

$$
\|u\|_{L^{p, \infty}\left(0, T ; L^{q, \infty}\left(\mathbb{R}^{3}\right)\right)} \leq \varepsilon, \quad \text { with } 2 / p+3 / q=1, \quad q>3 ;
$$

(2) $u \in L^{p}\left(0, T ; L^{\vec{q}}\left(\mathbb{R}^{3}\right)\right)$ with $2 / p+1 / q_{1}+1 / q_{2}+1 / q_{3}=2, \quad 1<q_{i}<\infty$;

(3) $\nabla u \in L^{2}\left(0, T ; L^{3}\left(\mathbb{R}^{3}\right)\right)$. 
Remark 1.5. Theorem 1.4 is an improvement of (1.21). In addition, collecting (1.21), (3) in Theorem 1.4 and Gagliardo-Nirenberg inequality, we obtain an alternative condition of (1.21) below

$$
\limsup _{t \rightarrow T^{*}}\|\nabla u\|_{L^{p}\left(0, t ; L^{q}\left(\mathbb{R}^{3}\right)\right)}=\infty, \quad \text { with } \frac{2}{p}+\frac{3}{q}=2,3 / 2<q \leq 3 .
$$

Remark 1.6. Very recently, Wang [36] proved (1.21) is also valid for nonhomogeneous incompressible Navier-Stokes equations with heat conducting. It is worth remarking that Theorem 1.4 also holds for this system. We leave this to the interested readers.

The remaining part of this paper is organized as follows. In the next section, some materials involving Lorentz spaces and anisotropic Lebesgue spaces are listed. Several auxiliary lemmas are also given. Then, we recall the local well-posedness of equations (1.1) and (1.20), respectively. Section 3 is devoted to proving Theorem 1.1 and Theorem 1.3. In Section 4, we are concerned with lifespan of nonhomogeneous incompressible Navier-Stokes equations.

\section{Preliminaries}

First, we introduce some notations used in this paper. For $p \in[1, \infty]$, the notation $L^{p}(0, T ; X)$ stands for the set of measurable functions $f(x, t)$ on the interval $(0, T)$ with values in $X$ and $\|f(\cdot, t)\|_{X}$ belonging to $L^{p}(0, T)$. The classical Sobolev space $W^{k, p}\left(\mathbb{R}^{3}\right)$ is

equipped with the norm $\|f\|_{W^{k, p}\left(\mathbb{R}^{3}\right)}=\sum_{|\alpha|=0}^{k}\left\|D^{\alpha} f\right\|_{L^{p}\left(\mathbb{R}^{3}\right)} \cdot|E|$ represents the $n$-dimensional Lebesgue measure of a set $E \subset \mathbb{R}^{n}$. We will use the summation convention on repeated indices. $C$ is an absolute constant which may be different from line to line unless otherwise stated in this paper.

A function $f$ belongs to the homogeneous Sobolev spaces $D^{k, l}\left(\mathbb{R}^{3}\right)$ if $u \in L_{\text {loc }}^{1}\left(\mathbb{R}^{3}\right)$ such that $\left\|\nabla^{k} u\right\|_{L^{l}\left(\mathbb{R}^{3}\right)}<\infty$. For simplicity, we write

$$
H^{k}=W^{k, 2}\left(\mathbb{R}^{3}\right), D^{k}=D^{k, 2}\left(\mathbb{R}^{3}\right) .
$$

We denote $G$ as the effective viscous flux, that is,

$$
G=(2 \mu+\lambda) \operatorname{div} u-P .
$$

The notation $\dot{v}=v_{t}+u \cdot \nabla v$ stands for material derivative. Note that

$$
\Delta G=\operatorname{div}(\rho \dot{u}) \quad \text { and } \quad-\mu \Delta(\operatorname{curl} u)=\nabla \times(\rho \dot{u}) .
$$

It is well-known that

$$
\|\nabla G\|_{L^{p}} \leq C\|\rho \dot{u}\|_{L^{p}},\|\nabla \operatorname{curl} u\|_{L^{p}} \leq C\|\rho \dot{u}\|_{L^{p}}, \forall p \in(1, \infty) .
$$




\subsection{Some basic facts on Lorentz spaces}

Next, we present some basic facts on Lorentz spaces. For $p, q \in[1, \infty]$, we define

$$
\|f\|_{L^{p, q}\left(\mathbb{R}^{3}\right)}=\left\{\begin{array}{l}
\left(p \int_{0}^{\infty} \alpha^{q}\left|\left\{x \in \mathbb{R}^{3}:|f(x)|>\alpha\right\}\right|^{\frac{q}{p}} \frac{d \alpha}{\alpha}\right)^{\frac{1}{q}}, \quad q<\infty, \\
\sup _{\alpha>0} \alpha\left|\left\{x \in \mathbb{R}^{3}:|f(x)|>\alpha\right\}\right|^{\frac{1}{p}}, \quad q=\infty .
\end{array}\right.
$$

Furthermore,

$$
L^{p, q}\left(\mathbb{R}^{3}\right)=\left\{f: f \text { is a measurable function on } \mathbb{R}^{3} \text { and }\|f\|_{L^{p, q}\left(\mathbb{R}^{3}\right)}<\infty\right\} .
$$

Similarly, one can define Lorentz spaces $L^{p, q}(0, T ; X)$ in time for $p \leq q \leq \infty$. $f \in$ $L^{p, q}(0, T ; X)$ means that $\|f\|_{L^{p, q}(0, T ; X)}<\infty$, where

$$
\|f\|_{L^{p, q}(0, T ; X)}=\left\{\begin{array}{l}
\left(p \int_{0}^{\infty} \alpha^{q}\left|\left\{t \in(0, T):\|f(t)\|_{X}>\alpha\right\}\right|^{\frac{q}{p}} \frac{d \alpha}{\alpha}\right)^{\frac{1}{q}}, \quad q<\infty \\
\sup _{\alpha>0} \alpha\left|\left\{t \in(0, T):\|f(t)\|_{X}>\alpha\right\}\right|^{\frac{1}{p}}, \quad q=\infty
\end{array}\right.
$$

We list the properties of Lorentz spaces as follows.

- Hölder's inequality in Lorentz spaces 25]

$$
\begin{aligned}
& \|f g\|_{L^{r, s}\left(\mathbb{R}^{n}\right)} \leq\|f\|_{L^{r_{1}, s_{1}\left(\mathbb{R}^{n}\right)}}\|g\|_{L^{r_{2}, s_{2}\left(\mathbb{R}^{n}\right)}}, \\
& \frac{1}{r}=\frac{1}{r_{1}}+\frac{1}{r_{2}}, \frac{1}{s}=\frac{1}{s_{1}}+\frac{1}{s_{2}} \text {. }
\end{aligned}
$$

- Interpolation characteristic of Lorentz spaces [2]

$$
\left(L^{p_{0}, q_{0}}\left(\mathbb{R}^{n}\right), L^{p_{1}, q_{1}}\left(\mathbb{R}^{n}\right)\right)_{\alpha, q}=L^{p, q}\left(\mathbb{R}^{n}\right) \quad \text { with } \quad \frac{1}{p}=\frac{1-\alpha}{p_{0}}+\frac{\alpha}{p_{1}}, 0<\alpha<1 .
$$

- Boundedness of Riesz Transform in Lorentz spaces [4]

$$
\left\|R_{j} f\right\|_{L^{p, q\left(\mathbb{R}^{n}\right)}} \leq C\|f\|_{L^{p, q}\left(\mathbb{R}^{n}\right)}, 1<p<\infty .
$$

- The Lorentz spaces $L^{p, r}$ increase as the exponent $r$ increases [13, 24]

For $1 \leq p \leq \infty$ and $1 \leq r_{1}<r_{2} \leq \infty$,

$$
\|f\|_{L^{p, r_{2}\left(\mathbb{R}^{n}\right)}} \leq\left(\frac{r_{1}}{p}\right)^{\frac{1}{r_{1}}-\frac{1}{r_{2}}}\|f\|_{L^{p, r_{1}\left(\mathbb{R}^{n}\right)}} .
$$

- Sobolev's inequality in Lorentz spaces [25, 33]

$$
\|f\|_{L^{\frac{n p}{n-p}, p}\left(\mathbb{R}^{n}\right)} \leq C\|\nabla f\|_{L^{p}\left(\mathbb{R}^{n}\right)} \text { with } 1 \leq p<n .
$$




\subsection{Some materials on anisotropic Lebesgue spaces}

Let $x=\left(x_{1}, x_{2}, x_{3}\right) \in \mathbb{R}^{3}, \vec{q}=\left(q_{1}, q_{2}, q_{3}\right)$, where $q_{i} \in[1, \infty], 1 \leq i \leq 3$. A function $f(x)$ belongs to the anisotropic Lebesgue space $L^{\vec{q}}\left(\mathbb{R}^{3}\right)$ if

$$
\|f\|_{L^{\vec{q}}\left(\mathbb{R}^{3}\right)}=\|f\|_{L_{1}^{q_{1}} L_{2}^{q_{2}} L_{3}^{q_{3}}\left(\mathbb{R}^{3}\right)}=\|\|\|f\|_{L_{x_{1}}^{q_{1}}(\mathbb{R})}\left\|_{L_{x_{2}}^{q_{2}(\mathbb{R})}}\right\|_{L_{x_{3}}^{q_{3}(\mathbb{R})}}<\infty .
$$

The study of anisotropic Lebesgue spaces first appears in Benedek and Panzone [1] .

We list the properties of anisotropic Lebesgue spaces as follows.

- The Hölder inequality in anisotropic Lebesgue spaces [1]

$$
\|f g\|_{L^{1}\left(\mathbb{R}^{3}\right)} \leq\|f\|_{L^{\vec{r}}\left(\mathbb{R}^{3}\right)}\|g\|_{L^{\vec{s}}\left(\mathbb{R}^{3}\right)} \text { with } \frac{1}{\vec{r}}+\frac{1}{\vec{s}}=1 .
$$

- The interpolation inequality in anisotropic Lebesgue spaces [1]

$$
\|f\|_{L^{\vec{r}}\left(\mathbb{R}^{3}\right)} \leq\|f\|_{L^{\vec{s}}\left(\mathbb{R}^{3}\right)}^{\alpha}\|f\|_{L^{\vec{t}}\left(\mathbb{R}^{3}\right)}^{1-\alpha} \text { with } \frac{1}{\vec{r}}=\frac{\alpha}{\vec{s}}+\frac{1-\alpha}{\vec{t}}, 0<\alpha<1 .
$$

- Boundedness of singular integral operator in anisotropic Lebesgue spaces 26]

$$
\left\|R_{j} f\right\|_{L^{\vec{q}\left(\mathbb{R}^{3}\right)}} \leq C\|f\|_{L^{\vec{q}}\left(\mathbb{R}^{3}\right)}, 1<\vec{q}<\infty .
$$

- Sobolev's inequality in anisotropic Lebesgue spaces [11, 39]

For $q_{1}, q_{2}, q_{3} \in[2, \infty)$ and $0 \leq \sum_{i} \frac{1}{q_{i}}-\frac{1}{2} \leq 1$,

$$
\begin{aligned}
\|f\|_{L^{\vec{q}}\left(\mathbb{R}^{3}\right)} & \leq C\left\|\partial_{1} f\right\|_{L^{2}\left(\mathbb{R}^{3}\right)}^{\frac{q_{1}-2}{2 q_{1}}}\left\|\partial_{2} f\right\|_{L^{2}\left(\mathbb{R}^{3}\right)}^{\frac{q_{2}-2}{2 q_{2}}}\left\|\partial_{3} f\right\|_{L^{2}\left(\mathbb{R}^{3}\right)}^{\frac{q_{3}-2}{2 q_{3}}}\|f\|_{L^{2}\left(\mathbb{R}^{3}\right)}^{\sum_{i} \frac{1}{q_{i}}-\frac{1}{2}} \\
& \leq C\|\nabla f\|_{L^{2}\left(\mathbb{R}^{3}\right)}^{\frac{3}{2}-\sum_{i} \frac{1}{q_{i}}}\|f\|_{L^{2}\left(\mathbb{R}^{3}\right)}^{\sum_{i} \frac{1}{q_{i}}-\frac{1}{2}}
\end{aligned}
$$

\subsection{Auxiliary lemmas}

The following powerful Gronwall lemma obtained by Bosia, Pata and Robinson in [3] helps us to prove Theorem 1.1.

Lemma 2.1 ([3]). Let $\phi$ be a measurable positive function defined on the interval $[0, T]$. Suppose that there exists $\tau_{0}>0$ such that for all $0<\tau<\tau_{0}$ and a.e. $t \in[0, T], \phi$ satisfies the inequality

$$
\frac{d}{d t} \phi \leq \mu \lambda^{1-\tau} \phi^{1+2 \tau}
$$

where $0<\lambda \in L^{1, \infty}(0, T)$ and $\mu>0$ with

$$
\mu\|\lambda\|_{L^{1, \infty}(0, T)}<\frac{1}{2}
$$

Then $\phi$ is bounded on $[0, T]$.

To apply the above lemma, we require the following fact. 
Lemma $2.2([19])$. Assume that the pair $(p, q)$ satisfies $\frac{2}{p}+\frac{3}{q}=a$ with $a, q \geq 1$ and $p>0$. Then, for every $\tau \in[0,1]$ and given $b, c_{0} \geq 1$, there exist $p_{\tau}>0$ and $\min \{q, b\} \leq q_{\tau} \leq$ $\max \{q, b\}$ such that

$$
\left\{\begin{array}{l}
\frac{2}{p_{\tau}}+\frac{3}{q_{\tau}}=a, \\
\frac{p_{\tau}}{q_{\tau}}=\frac{p(1-\tau)}{q}+\frac{c_{0} \tau}{b} .
\end{array}\right.
$$

Finally, we recall the local well-posedness of strong solutions to the full compressible Navier-Stokes equations (1.1) and nonhomogeneous incompressible Navier-Stokes equations (1.20).

Proposition $2.3([8])$. Suppose $u_{0}, \theta_{0} \in D^{1}\left(\mathbb{R}^{3}\right) \cap D^{2}\left(\mathbb{R}^{3}\right)$ and

$$
\rho_{0} \in W^{1, q}\left(\mathbb{R}^{3}\right) \cap H^{1}\left(\mathbb{R}^{3}\right) \cap L^{1}\left(\mathbb{R}^{3}\right)
$$

for some $q \in(3,6]$. If $\rho_{0}$ is nonnegative and the initial data to (1.1) satisfy the compatibility condition

$$
\begin{aligned}
& -\mu \Delta u_{0}-(\mu+\lambda) \nabla \operatorname{div} u_{0}+\nabla P\left(\rho_{0}, \theta_{0}\right)=\sqrt{\rho_{0}} g_{1} \\
& \Delta \theta_{0}+\frac{\mu}{2}\left|\nabla u_{0}+\left(\nabla u_{0}\right)^{t r}\right|^{2}+\lambda\left(\operatorname{div} u_{0}\right)^{2}=\sqrt{\rho_{0}} g_{2}
\end{aligned}
$$

for vector fields $g_{1}, g_{2} \in L^{2}\left(\mathbb{R}^{3}\right)$. Then there exist $T \in(0, \infty]$ and a unique solution to (1.1), satisfying

$$
\begin{aligned}
& (\rho, u, \theta) \in C\left([0, T) ; L^{1} \cap H^{1}\left(\mathbb{R}^{3}\right) \cap W^{1, q}\left(\mathbb{R}^{3}\right)\right) \times C\left([0, T) ; D^{1} \cap D^{2}\right) \times L^{2}\left([0, T) ; D^{2, q}\right) \\
& \left(\rho_{t}, u_{t}, \theta_{t}\right) \in C\left([0, T) ; L^{2} \cap L^{q}\left(\mathbb{R}^{3}\right)\right) \times L^{2}\left([0, T) ; D^{1}\right) \times L^{2}\left([0, T) ; D^{1}\right) \\
& \left(\rho^{1 / 2} u_{t}, \rho^{1 / 2} \theta_{t}\right) \in L^{\infty}\left([0, T) ; L^{2}\left(\mathbb{R}^{3}\right)\right) \times L^{\infty}\left([0, T) ; L^{2}\right) .
\end{aligned}
$$

Proposition 2.4 ([6] $]$ ). Suppose $u_{0} \in D^{1}\left(\mathbb{R}^{3}\right) \cap D^{2}\left(\mathbb{R}^{3}\right)$ and

$$
\rho_{0} \in W^{1, q}\left(\mathbb{R}^{3}\right) \cap H^{1}\left(\mathbb{R}^{3}\right) \cap L^{1}\left(\mathbb{R}^{3}\right)
$$

for some $q \in(3,6]$. If $\rho_{0}$ is nonnegative and the initial data to (1.20) satisfy the compatibility condition

$$
-\Delta u_{0}+\nabla \Pi_{0}=\sqrt{\rho_{0}} g
$$

for vector field $g \in L^{2}\left(\mathbb{R}^{3}\right)$. Then there exist $T \in(0, \infty]$ and a unique solution to (1.20), satisfying

$$
\begin{aligned}
& (\rho, u) \in C\left([0, T) ; L^{1}\left(\mathbb{R}^{3}\right) \cap H^{1}\left(\mathbb{R}^{3}\right) \cap W^{1, q}\left(\mathbb{R}^{3}\right)\right) \times C\left([0, T) ; D^{1} \cap D^{2}\right) \\
& \left(\rho_{t}, u_{t}\right) \in C\left([0, T) ; L^{2}\left(\mathbb{R}^{3}\right) \cap L^{q}\left(\mathbb{R}^{3}\right)\right) \times L^{2}\left([0, T) ; D^{1}\right) \\
& \rho^{1 / 2} u_{t} \in L^{\infty}\left([0, T) ; L^{2}\left(\mathbb{R}^{3}\right)\right)
\end{aligned}
$$

\section{$3 \quad$ Full compressible Navier-Stokes equations}

\subsection{Continuation criteria in Lorentz spaces}

This section is devoted to proving Theorem 1.1. 
Proof of (10) in Theorem 1.1. Multiplying $(1.1)_{2}$ by $u_{t}$, integrating over $\mathbb{R}^{3}$, we can write

$$
\begin{aligned}
& \frac{1}{2} \frac{d}{d t} \int\left[\mu|\nabla u|^{2}+(\lambda+\mu)(\operatorname{div} u)^{2}\right] d x+\int \rho|\dot{u}|^{2} d x \\
= & \int \rho \dot{u} \cdot(u \cdot \nabla u) d x+\int P \operatorname{div} u_{t} d x \\
= & I+I I .
\end{aligned}
$$

In view of the Young inequality, we see that

$$
I \leq \frac{1}{4} \int \rho|\dot{u}|^{2} d x+C \int|u|^{2}|\nabla u|^{2} d x .
$$

By means of the effective viscous flux (2.1), we calculate

$$
\begin{aligned}
I I & =\frac{d}{d t} \int P \operatorname{div} u d x-\int P_{t} \operatorname{div} u d x \\
& =\frac{d}{d t} \int P \operatorname{div} u-\frac{1}{2(2 \mu+\lambda)} \frac{d}{d t} \int P^{2}-\frac{1}{2 \mu+\lambda} \int P_{t} G \\
& =I I_{1}+I I_{2}+I I_{3} .
\end{aligned}
$$

We define total energy

$$
E=: \theta+\frac{|u|^{2}}{2}
$$

It is clear that, for $\alpha \geq 1$,

$$
0 \leq \theta^{\alpha} \leq E^{\alpha}
$$

From (1.1), we know that

$$
(\rho E)_{t}-\frac{\kappa}{c_{v}} \operatorname{div}(\nabla E)=\operatorname{div} F
$$

where

$$
F=-\rho E u+\frac{\mu-\kappa c_{v}}{2} \nabla\left(|u|^{2}\right)+\mu u \cdot \nabla u+\lambda u \operatorname{div} u-P u .
$$

Performing some direct calculations yields that

$$
\begin{aligned}
|F| & \leq C(|\rho E u|+|u||\nabla u|+|P u|) \\
& \leq C(|\rho E u|+|u||\nabla u|+R|\rho \theta u|) \\
& \leq C(|\rho E u|+|u||\nabla u|) .
\end{aligned}
$$

According to $P=R \rho \theta=R\left(\rho E-\rho \frac{|u|^{2}}{2}\right)$ and (3.5), we note that

$$
\begin{aligned}
I I_{3} & =-\frac{R}{2 \mu+\lambda} \int(\rho E)_{t} G+\frac{1}{2 \mu+\lambda} \int\left(\frac{\rho|u|^{2}}{2}\right)_{t} G \\
& =-\frac{R}{2 \mu+\lambda} \int \operatorname{div}\left(F+\frac{\kappa}{c_{v}} \nabla E\right) G+\frac{R}{2 \mu+\lambda} \int\left(\frac{\rho|u|^{2}}{2}\right)_{t} G \\
& =\frac{R}{2 \mu+\lambda} \int\left(F+\frac{\kappa}{c_{v}} \nabla E\right) \nabla G+\frac{R}{2 \mu+\lambda} \int\left(\frac{\rho|u|^{2}}{2}\right)_{t} G \\
& =I I_{31}+I I_{32} .
\end{aligned}
$$


In light of (3.6), the Young inequality and (2.2), we have

$$
\begin{aligned}
I I_{31} & \leq C \int(|F|+|\nabla E|)|\nabla G| \\
& \leq C \int(|\rho E u|+|u||\nabla u|+|\nabla E|) \nabla G \\
& \leq \eta\|\nabla G\|_{L^{2}}^{2}+C \int\left(\rho^{2} E^{2}|u|^{2}+|\nabla E|^{2}+|u|^{2}|\nabla u|^{2}\right) d x \\
& \leq \eta C\|\rho \dot{u}\|_{L^{2}}^{2}+C \int\left(\rho^{2} E^{2}|u|^{2}+|\nabla E|^{2}+|u|^{2}|\nabla u|^{2}\right) d x .
\end{aligned}
$$

It is straightforward to check

$$
\begin{aligned}
I I_{32} & =\frac{R}{2 \mu+\lambda} \int \frac{\rho_{t}|u|^{2}}{2} G+\frac{R}{2 \mu+\lambda} \int \rho u \cdot u_{t} G \\
& =I I_{321}+I I_{322} .
\end{aligned}
$$

Making use of $\rho_{t}=-\operatorname{div}(\rho u)$, integrating by parts, the Young inequality, (3.4) and (2.2), we see that

$$
\begin{aligned}
I I_{321} & =-\frac{R}{2 \mu+\lambda} \int \frac{\operatorname{div}(\rho u)|u|^{2}}{2} G \\
& =\frac{R}{2 \mu+\lambda} \int \rho u \cdot \nabla u \cdot u G+\frac{R}{2 \mu+\lambda} \int \frac{\rho u|u|^{2}}{2} \cdot \nabla G \\
& \leq C \int \rho|u \cdot \nabla u|^{2}+C \int \rho|u|^{2}|G|^{2}+C \int \rho|u|^{6}+\eta \int|\nabla G|^{2} \\
& \leq C \int \rho|u \cdot \nabla u|^{2}+C \int \rho|u|^{2}\left(|\nabla u|^{2}+R \rho^{2} \theta^{2}\right)+C \int \rho|u|^{2}\left(\theta^{2}+E^{2}\right)+\eta C \int|\rho \dot{u}|^{2} \\
& \leq C \int \rho|u|^{2}|\nabla u|^{2}+C \int \rho|u|^{2} E^{2}+\eta C \int|\rho \dot{u}|^{2},
\end{aligned}
$$

where we have used the fact

$$
|G| \leq C(|\nabla u|+|\theta \rho|) \leq C(|\nabla u|+|E \rho|) .
$$

Utilizing $\dot{u}=u_{t}+u \cdot \nabla u$, the Young inequality, and (3.11), we arrive at that

$$
\begin{aligned}
I I_{322} & =\frac{R}{2 \mu+\lambda} \int \rho u \cdot(\dot{u}-u \cdot \nabla u) G \\
& =\frac{R}{2 \mu+\lambda} \int \rho u \cdot \dot{u} G-\int \rho u u \cdot \nabla u G \\
& \leq \eta \int \rho|\dot{u}|^{2} d x+C \int \rho|u|^{2}|G|^{2} d x+\int \rho|u|^{2}|\nabla u|^{2} \\
& \leq \eta \int \rho|\dot{u}|^{2} d x+C \int \rho|u|^{2}|\nabla u|^{2}+C \int \rho|u|^{2} E^{2} .
\end{aligned}
$$

Plugging (3.10) and (3.12) into (3.9), we deduce

$$
I I_{32} \leq \eta(1+C) \int \rho|\dot{u}|^{2} d x+C \int|u|^{2}|\nabla u|^{2}+C \int \rho|u|^{2} E^{2} .
$$


It follows from (3.7), (3.8) and (3.13) that

$$
I I_{3} \leq C \eta \int \rho|\dot{u}|^{2} d x+C \int \rho|u|^{2}|\theta|^{2} d x+C \int|u|^{2}|\nabla u|^{2}+C \int|\nabla E|^{2} .
$$

Combining (3.1), (3.2), (3.3) and (3.14) and choosing $\eta$ sufficiently small lead us to the estimate

$$
\begin{aligned}
& \frac{1}{2} \frac{d}{d t} \int\left[\mu|\nabla u|^{2}+(\lambda+\mu)(\operatorname{div} u)^{2}+\frac{1}{(2 \mu+\lambda)} P^{2}-2 P \operatorname{div} u\right] d x+\frac{1}{2} \int \rho|\dot{u}|^{2} d x \\
& \leq C_{1} \int \rho|u|^{2}|E|^{2} d x+C_{1} \int|u|^{2}|\nabla u|^{2}+C_{1} \int|\nabla E|^{2} .
\end{aligned}
$$

To control the first term of right hand side of the latter relation, we use (3.5). More precisely, multiplying (3.5) by $E$, integrating by parts and invoking $(\rho E)_{t} E=\frac{1}{2}\left(\rho E^{2}\right)_{t}-\frac{1}{2} \operatorname{div}(\rho u)$, we find

$$
\begin{aligned}
\frac{1}{2} \frac{d}{d t} \int \rho E^{2}+\frac{\kappa}{c_{v}} \int|\nabla E|^{2} d x & =\frac{1}{2} \int \operatorname{div}(\rho u) E^{2}+\int E \operatorname{div} F \\
& =-\frac{1}{2} \int(\rho u) \nabla E^{2}-\int F \nabla E .
\end{aligned}
$$

The Young inequality helps us to obtain

$$
\begin{aligned}
& -\frac{1}{2} \int(\rho u) \nabla E^{2} \leq C \int \rho|u|^{2}|E|^{2}+\frac{\kappa}{4 c_{v}} \int|\nabla E|^{2} d x, \\
& \int F \nabla E \leq C \int|F|^{2}+\frac{\kappa}{4 c_{v}} \int|\nabla E|^{2} d x .
\end{aligned}
$$

As a consequence,

$$
\begin{aligned}
\frac{1}{2} \frac{d}{d t} \int \rho E^{2}+\frac{\kappa}{2 c_{v}} \int|\nabla E|^{2} d x & \leq C \int \rho|u|^{2}|E|^{2}+C \int|F|^{2} \\
& \leq C \int|u|^{2}|\nabla u|^{2} d x+C \int \rho|u|^{2}|E|^{2} .
\end{aligned}
$$

Multiplying both sides of (3.17) by $\frac{c_{v}\left(2 C_{1}+C_{2}\right)}{\kappa}$ and adding it to (3.15), we know that

$$
\frac{d}{d t} \Phi(t)+\frac{1}{2} \int \rho|\dot{u}|^{2} d x+\frac{C_{2}}{2} \int|\nabla E|^{2} \leq C \int \rho|u|^{2}|E|^{2} d x+C \int|u|^{2}|\nabla u|^{2},
$$

where

$$
\Phi(t)=\frac{1}{2} \int\left[\mu|\nabla u|^{2}+(\lambda+\mu)(\operatorname{div} u)^{2}+\frac{1}{(2 \mu+\lambda)} P^{2}-2 P \operatorname{div} u+\frac{c_{v}\left(2 C_{1}+C_{2}\right)}{\kappa} \rho E^{2}\right] d x .
$$

According to $P=R \rho \theta$, we can choose $C_{2}$ sufficiently large to make sure that

$$
\mu|\nabla u|^{2}+\frac{c_{v}\left(2 C_{1}+C_{2}\right)}{\kappa} \rho E^{2}-2 P \operatorname{div} u \geq \frac{\mu}{2}|\nabla u|^{2}+\rho E^{2} .
$$

Thus, $\Phi(t) \geq 0$. 
With the help of the Hölder inequality (2.3) or interpolation characteristic (2.4) and Sobolev embedding (2.7) in Lorentz spaces, we get

$$
\begin{aligned}
\left\|\rho E^{2}\right\|_{L^{\frac{q}{q-2}, 1}\left(\mathbb{R}^{3}\right)} & \leq\|\sqrt{\rho} E\|_{L^{6,2}\left(\mathbb{R}^{3}\right)}^{\frac{6}{q}}\|\sqrt{\rho} E\|_{L^{2}\left(\mathbb{R}^{3}\right)}^{2-\frac{6}{q}} \\
& \leq C\|E\|_{L^{6,2}\left(\mathbb{R}^{3}\right)}^{\frac{6}{q}}\|\sqrt{\rho} E\|_{L^{2}\left(\mathbb{R}^{3}\right)}^{2-\frac{6}{q}} \\
& \leq C\|\nabla E\|_{L^{2}\left(\mathbb{R}^{3}\right)}^{\frac{6}{q}}\|\sqrt{\rho} E\|_{L^{2}\left(\mathbb{R}^{3}\right)}^{2-\frac{6}{4}} .
\end{aligned}
$$

Therefore, Hölder's inequality (2.3), (3.20) and the Young inequality imply that

$$
\begin{aligned}
\int \rho|u|^{2}|E|^{2} d x & \leq\left\||u|^{2}\right\|_{L^{\frac{q}{2}, \infty}\left(\mathbb{R}^{3}\right)}\left\|\rho E^{2}\right\|_{L^{\frac{q}{q-2}, 1}\left(\mathbb{R}^{3}\right)} \\
& \leq\|u\|_{L^{\frac{q}{2}, \infty}\left(\mathbb{R}^{3}\right)}^{2}\|\sqrt{\rho} E\|_{L^{\frac{2 q}{q-2}, 2}\left(\mathbb{R}^{3}\right)}^{2} \\
& \leq C\|u\|_{L^{q, \infty}\left(\mathbb{R}^{3}\right)}^{2}\|\nabla E\|_{L^{2}\left(\mathbb{R}^{3}\right)}^{\frac{6}{q}}\|\sqrt{\rho} E\|_{L^{2}\left(\mathbb{R}^{3}\right)}^{2-\frac{6}{q}} \\
& \leq C\|u\|_{L^{q, \infty}\left(\mathbb{R}^{3}\right)}^{\frac{2 q}{q-3}}\|\sqrt{\rho} E\|_{L^{2}\left(\mathbb{R}^{3}\right)}^{2}+\frac{C_{2}}{8}\|\nabla E\|_{L^{2}\left(\mathbb{R}^{3}\right)}^{2} .
\end{aligned}
$$

Employing boundedness of Riesz Transform in Lorentz spaces (2.5), (2.1), Sobolev inequality (2.7) in Lorentz spaces and (2.2), we infer that

$$
\begin{aligned}
\|\nabla u\|_{L^{6,2}\left(\mathbb{R}^{3}\right)} & \leq C\left(\|\operatorname{div} u\|_{L^{6,2}\left(\mathbb{R}^{3}\right)}+\|\operatorname{curl} u\|_{L^{6,2}\left(\mathbb{R}^{3}\right)}\right) \\
& \leq C\left(\|G\|_{L^{6,2}\left(\mathbb{R}^{3}\right)}+\|P\|_{L^{6,2}\left(\mathbb{R}^{3}\right)}+\|\operatorname{curl} u\|_{L^{6,2}\left(\mathbb{R}^{3}\right)}\right) \\
& \leq C\left(\|G\|_{L^{6,2}\left(\mathbb{R}^{3}\right)}+\|\rho E\|_{L^{6,2}\left(\mathbb{R}^{3}\right)}+\|\operatorname{curl} u\|_{L^{6,2}\left(\mathbb{R}^{3}\right)}\right) \\
& \leq C\left(\|\nabla G\|_{L^{2,2}\left(\mathbb{R}^{3}\right)}+\|\nabla E\|_{L^{2,2}\left(\mathbb{R}^{3}\right)}+\|\nabla \operatorname{curl} u\|_{L^{2,2}\left(\mathbb{R}^{3}\right)}\right) \\
& \leq C\left(\|\rho \dot{u}\|_{L^{2}\left(\mathbb{R}^{3}\right)}+\|\nabla E\|_{L^{2}\left(\mathbb{R}^{3}\right)}\right) .
\end{aligned}
$$

With this in hand, modifying the proof of (3.21), we conclude that

$$
\begin{aligned}
C \int|u|^{2}|\nabla u|^{2} & \leq\left\|\left.u\right|^{2}\right\|_{L^{\frac{q}{2}, \infty}\left(\mathbb{R}^{3}\right)}\left\||\nabla u|^{2}\right\|_{L^{\frac{q}{q-2}, 1}\left(\mathbb{R}^{3}\right)} \\
& \leq\|u\|_{L^{q, \infty}\left(\mathbb{R}^{3}\right)}^{2}\|\nabla u\|_{L^{\frac{2 q}{q-2}, 2}\left(\mathbb{R}^{3}\right)}^{2} \\
& \leq C\|u\|_{L^{q, \infty}\left(\mathbb{R}^{3}\right)}^{2}\|\nabla u\|_{L^{2}\left(\mathbb{R}^{3}\right)}^{2-\frac{6}{q}}\|\nabla u\|_{L^{6,2}\left(\mathbb{R}^{3}\right)}^{\frac{6}{q}} \\
& \leq C\|u\|_{L^{q, \infty}\left(\mathbb{R}^{3}\right)}^{2}\|\nabla u\|_{L^{2}\left(\mathbb{R}^{3}\right)}^{2-\frac{6}{q}}\left(\|\rho \dot{u}\|_{L^{2}\left(\mathbb{R}^{3}\right)}+\|\nabla E\|_{L^{2}\left(\mathbb{R}^{3}\right)}\right)^{\frac{6}{q}} \\
& \leq C\|u\|_{L^{q, \infty}\left(\mathbb{R}^{3}\right)}^{\frac{2 q}{q-3}}\|\nabla u\|_{L^{2}\left(\mathbb{R}^{3}\right)}^{2}+\frac{1}{4}\|\rho \dot{u}\|_{L^{2}\left(\mathbb{R}^{3}\right)}+\frac{C_{2}}{8}\|\nabla E\|_{L^{2}\left(\mathbb{R}^{3}\right)}
\end{aligned}
$$

Substituting (3.21) and (3.22) into (3.18), we have

$$
\begin{aligned}
& \frac{d}{d t} \Phi(t)+\frac{1}{4} \int \rho|\dot{u}|^{2} d x+\frac{C_{2}}{4} \int|\nabla E|^{2} d x \\
\leq & C\|u\|_{L^{q, \infty}\left(\mathbb{R}^{3}\right)}^{\frac{2 q}{q-3}}\left(\|\sqrt{\rho} E\|_{L^{2}\left(\mathbb{R}^{3}\right)}^{2}+\|\nabla u\|_{L^{2}\left(\mathbb{R}^{3}\right)}^{2}\right) .
\end{aligned}
$$

Owing to (3.19) and dropping the unnecessary terms, we infer

$$
\frac{d}{d t} \Phi(t) \leq C\|u\|_{L^{q, \infty}\left(\mathbb{R}^{3}\right)}^{\frac{2 q}{q-3}} \Phi(t) .
$$


The interpolation characteristic (2.4), Sobolev's embedding inequality (2.7), Lemma 2.2 with $a=1, b=6, c_{0}=4$ and (2.6) allow us to deduce the estimate

$$
\begin{aligned}
\|u\|_{L^{q \tau}, \infty\left(\mathbb{R}^{3}\right)}^{p_{\tau}} & \leq\|u\|_{L^{q, \infty}\left(\mathbb{R}^{3}\right)}^{p(1-\tau)}\|u\|_{L^{6, \infty}\left(\mathbb{R}^{3}\right)}^{4 \tau} \\
& \leq C\|u\|_{L^{q, \infty}\left(\mathbb{R}^{3}\right)}^{p(1-\tau)}\|u\|_{L^{6,2}\left(\mathbb{R}^{3}\right)}^{4 \tau} \\
& \leq C\|u\|_{L^{q, \infty}\left(\mathbb{R}^{3}\right)}^{p(1-\tau)}\|\nabla u\|_{L^{2}\left(\mathbb{R}^{3}\right)}^{4 \tau} \\
& \leq C\|u\|_{L^{q, \infty}\left(\mathbb{R}^{3}\right)}^{p(1-\tau)} \Phi(t)^{2 \tau}
\end{aligned}
$$

where (3.19) was used again.

Since the pair $\left(p_{\tau}, q_{\tau}\right)$ also meets $2 / p_{\tau}+3 / q_{\tau}=1$, we insert (3.25) into (3.24) to obtain

$$
\frac{d}{d t} \Phi(t) \leq C\|u\|_{L^{q \tau}, \infty\left(\mathbb{R}^{3}\right)}^{p_{\tau}} \Phi(t) \leq C\|u\|_{L^{q, \infty\left(\mathbb{R}^{3}\right)}}^{p(1-\tau)} \Phi(t)^{1+2 \tau}
$$

At this stage, Lemma 2.1 is applicable. This together with (1.10) enables us to finish the proof of this part.

Proof of (2) in Theorem 1.1, Along the lines of [18], we see that

$$
\begin{aligned}
\frac{d}{d t} \Psi(t)+\frac{\kappa}{2} \int|\nabla \theta|^{2} d x+ & \frac{1}{2} \int \rho|\dot{u}|^{2} d x+\frac{1}{2} \int_{\mathbb{R}^{3} \cap\{|u|>0\}}|u|^{2}|\nabla u|^{2} \\
& \leq C\left(\int \rho|\theta|^{3} d x+\int \rho|u|^{2}|\theta|^{2} d x\right),
\end{aligned}
$$

where

$$
\Psi(t)=\int\left[\frac{\mu}{2}|\nabla u|^{2}+(\mu+\lambda)(\operatorname{div} u)^{2}+\frac{1}{2 \mu+\lambda} P^{2}-2 P \operatorname{div} u+\frac{C_{3} c_{\nu}}{2} \rho \theta^{2}+\frac{C_{4}+1}{2 \mu} \rho|u|^{4}\right] d x .
$$

To bound the right hand side of (3.26), we infer from the Hölder inequality (2.3) or interpolation characteristic (2.4) and Sobolev embedding (2.7) in Lorentz spaces that

$$
\begin{aligned}
\left\||u|^{2}\right\|_{L^{\frac{2 q}{q-1}, 2}\left(\mathbb{R}^{3}\right)} & \leq C\left\||u|^{2}\right\|_{L^{2}\left(\mathbb{R}^{3}\right)}^{1-\frac{3}{2 q}}\left\||u|^{2}\right\|_{L^{6,2}\left(\mathbb{R}^{3}\right)}^{\frac{3}{2 q}} \\
& \leq C\left\||u|^{2}\right\|_{L^{2}\left(\mathbb{R}^{3}\right)}^{1-\frac{3}{2 q}}\left\|\nabla|u|^{2}\right\|_{L^{2}\left(\mathbb{R}^{3}\right)}^{\frac{3}{2 q}}
\end{aligned}
$$

Likewise,

$$
\left\|\rho^{\frac{1}{2}} \theta\right\|_{L^{\frac{2 q}{q-1}, 2}\left(\mathbb{R}^{3}\right)} \leq C\|\theta\|_{L^{2}\left(\mathbb{R}^{3}\right)}^{1-\frac{3}{2 q}}\|\nabla \theta\|_{L^{2}\left(\mathbb{R}^{3}\right)}^{\frac{3}{2 q}}
$$


The Hölder's inequality (2.3), (3.27), (3.28) and the Young inequality show us that

$$
\begin{aligned}
\int \rho|u|^{2}|\theta|^{2} d x & =\int \rho^{\frac{1}{2}}|\theta| \rho^{\frac{1}{2}}|u|^{2}|\theta| d x \\
& \leq\|\theta\|_{L^{q, \infty}\left(\mathbb{R}^{3}\right)}\left\|\rho^{\frac{1}{2}} \theta\right\|_{L^{\frac{2 q}{q-1}, 2}\left(\mathbb{R}^{3}\right)}\left\|\rho^{\frac{1}{2}}|u|^{2}\right\|_{L^{\frac{2 q}{q-1}, 2}\left(\mathbb{R}^{3}\right)} \\
& \leq\|\theta\|_{L^{q, \infty}\left(\mathbb{R}^{3}\right)}\left\|\rho^{\frac{1}{2}} \theta\right\|_{L^{2}\left(\mathbb{R}^{3}\right)}^{1-\frac{3}{2 q}}\left\|\rho^{\frac{1}{2}} \theta\right\|_{L^{6}\left(\mathbb{R}^{3}\right)}^{\frac{3}{2 q}}\left\|\rho^{\frac{1}{2}}|u|^{2}\right\|_{L^{2}\left(\mathbb{R}^{3}\right)}^{1-\frac{3}{2 q}}\left\|\rho^{\frac{1}{2}}|u|^{2}\right\|_{L^{6}\left(\mathbb{R}^{3}\right)}^{\frac{3}{2 q}} \\
& \leq C\|\theta\|_{L^{q, \infty}\left(\mathbb{R}^{3}\right)}\left\|\rho^{\frac{1}{2}} \theta\right\|_{L^{2}\left(\mathbb{R}^{3}\right)}^{1-\frac{3}{2 q}}\left\|\rho^{\frac{1}{2}}|u|^{2}\right\|_{L^{2}\left(\mathbb{R}^{3}\right)}^{1-\frac{3}{2 q}}\left(\|\nabla \theta\|_{L^{2}\left(\mathbb{R}^{3}\right)}^{\frac{3}{q}}+\left\|\nabla|u|^{2}\right\|_{L^{2}\left(\mathbb{R}^{3}\right)}^{\frac{3}{q}}\right) \\
& \leq C\|\theta\|_{L^{q, \infty}\left(\mathbb{R}^{3}\right)}^{\frac{2 q}{2 q-3}}\left\|\rho^{\frac{1}{2}} \theta\right\|_{L^{2}\left(\mathbb{R}^{3}\right)}\left\|\rho^{\frac{1}{2}}|u|^{2}\right\|_{L^{2}\left(\mathbb{R}^{3}\right)}+\eta_{1}\|\nabla \theta\|_{L^{2}\left(\mathbb{R}^{3}\right)}^{2}+\eta_{2}\left\|\nabla|u|^{2}\right\|_{L^{2}\left(\mathbb{R}^{3}\right)}^{2} \\
& \leq C\|\theta\|_{L^{q, \infty}\left(\mathbb{R}^{3}\right)}^{\frac{2 q}{2-3}}\left(\left\|\rho^{\frac{1}{2}} \theta\right\|_{L^{2}\left(\mathbb{R}^{3}\right)}^{2}+\left\|\rho^{\frac{1}{2}}|u|^{2}\right\|_{L^{2}\left(\mathbb{R}^{3}\right)}^{2}\right)+\eta_{1}\|\nabla \theta\|_{L^{2}\left(\mathbb{R}^{3}\right)}^{2}+\eta_{2}\left\|\nabla|u|^{2}\right\|_{L^{2}\left(\mathbb{R}^{3}\right)}^{2}
\end{aligned}
$$

Arguing in the same manner as the above, we see that

$$
\begin{aligned}
\int \rho|\theta|^{3} d x & =\int \rho^{\frac{1}{2}}|\theta| \rho^{\frac{1}{2}} \theta|\theta| d x \\
& \leq\|\theta\|_{L^{q, \infty}\left(\mathbb{R}^{3}\right)}\left\|\rho^{\frac{1}{2}} \theta\right\|_{L^{\frac{2 q}{q-1}, 2}\left(\mathbb{R}^{3}\right)}^{2} \\
& \leq\|\theta\|_{L^{q, \infty}\left(\mathbb{R}^{3}\right)}\left\|\rho^{\frac{1}{2}} \theta\right\|_{L^{2}\left(\mathbb{R}^{3}\right)}^{2-\frac{3}{q}}\left\|\rho^{\frac{1}{2}} \theta\right\|_{L^{6,2}\left(\mathbb{R}^{3}\right)}^{\frac{3}{q}} \\
& \leq C\|\theta\|_{L^{q, \infty}\left(\mathbb{R}^{3}\right)}\left\|\rho^{\frac{1}{2}} \theta\right\|_{L^{2}\left(\mathbb{R}^{3}\right)}^{2-\frac{3}{q}}\|\theta\|_{L^{6,2}\left(\mathbb{R}^{3}\right)}^{\frac{3}{q}} \\
& \leq C\|\theta\|_{L^{q, \infty}\left(\mathbb{R}^{3}\right)}\left\|\rho^{\frac{1}{2}} \theta\right\|_{L^{2}\left(\mathbb{R}^{3}\right)}^{2-\frac{3}{q}}\|\nabla \theta\|_{L^{2}\left(\mathbb{R}^{3}\right)}^{\frac{3}{q}} \\
& \leq C\|\theta\|_{L^{q, \infty}\left(\mathbb{R}^{3}\right)}^{\frac{2 q}{2 q-3}}\left\|\rho^{\frac{1}{2}} \theta\right\|_{L^{2}\left(\mathbb{R}^{3}\right)}^{2}+\eta_{3}\|\nabla \theta\|_{L^{2}\left(\mathbb{R}^{3}\right)}^{2}
\end{aligned}
$$

Substituting (3.29) and (3.30) into (3.26), we have

$$
\begin{aligned}
\frac{d}{d t} \Psi(t)+\frac{\kappa}{4} \int|\nabla \theta|^{2} d x+ & \frac{1}{2} \int \rho|\dot{u}|^{2} d x+\frac{1}{4} \int_{\mathbb{R}^{3} \cap\{|u|>0\}}|u|^{2}|\nabla u|^{2} \\
& \leq C\|\theta\|_{L^{q, \infty}\left(\mathbb{R}^{3}\right)}^{\frac{2 q}{2 q-3}}\left(\left\|\rho^{\frac{1}{2}} \theta\right\|_{L^{2}\left(\mathbb{R}^{3}\right)}^{2}+\left\|\rho^{\frac{1}{2}}|u|^{2}\right\|_{L^{2}\left(\mathbb{R}^{3}\right)}^{2}\right) \\
& \leq C\|\theta\|_{L^{q, \infty}\left(\mathbb{R}^{3}\right)}^{\frac{2 q}{2 q-3}} \Psi(t),
\end{aligned}
$$

where we used the fact that

$$
\int(\mu+\lambda)(\operatorname{div} u)^{2}+\frac{1}{2 \mu+\lambda} P^{2}-2 P \operatorname{div} u+\frac{C_{3} C_{\nu}}{2} \rho \theta^{2} d x \geq \int \rho \theta^{2} d x,
$$

provided that the constant $C_{3}$ is suitable large enough.

Thanks to interpolation characteristic (2.4) or the Hölder inequality (2.3), applying lemma 2.2 with $a=2, b=6$ and (2.6) , we see that

$$
\|\theta\|_{L^{q \tau}, \infty\left(\mathbb{R}^{3}\right)}^{p_{\tau}} \leq\|\theta\|_{L^{q, \infty}\left(\mathbb{R}^{3}\right)}^{p(1-\tau)}\|\theta\|_{L^{6, \infty}\left(\mathbb{R}^{3}\right)}^{c_{0} \tau} \leq C\|\theta\|_{L^{q, \infty}\left(\mathbb{R}^{3}\right)}^{p(1-\tau)}\|\theta\|_{L^{6}\left(\mathbb{R}^{3}\right)}^{c_{0} \tau} \leq C\|\theta\|_{L^{q, \infty}\left(\mathbb{R}^{3}\right)}^{p(1-\tau)}\|\nabla \theta\|_{L^{2}\left(\mathbb{R}^{3}\right)}^{c_{0} \tau},
$$

where $c_{0}$ is a constant to be determined later. 
Since the pair $\left(p_{\tau}, q_{\tau}\right)$ also meets $2 / p_{\tau}+3 / q_{\tau}=2$, we insert (3.32) into (3.31) to obtain

$$
\begin{aligned}
\frac{d}{d t} \Psi(t)+\frac{\kappa}{4} \int|\nabla \theta|^{2} d x+ & \frac{1}{4} \int \rho|\dot{u}|^{2} d x+\frac{1}{4} \int_{\mathbb{R}^{3} \cap\{|u|>0\}}|u|^{2}|\nabla u|^{2} \\
& \leq C\|\theta\|_{L^{q, \infty\left(\mathbb{R}^{3}\right)}}^{p(1-\tau)}\|\nabla \theta\|_{L^{2}\left(\mathbb{R}^{3}\right)}^{c_{0} \tau} \Psi(t) \\
& \leq C\|\theta\|_{L^{q, \infty\left(\mathbb{R}^{3}\right)}}^{\frac{2 p(1-\tau)}{2-c_{0}}} \Psi(t)^{\frac{2}{2-c_{0} \tau}}+\frac{\kappa}{8}\|\nabla \theta\|_{L^{2}\left(\mathbb{R}^{3}\right)}^{2},
\end{aligned}
$$

Before going further, we take

$$
\delta=\frac{\left(2-c_{0}\right) \tau}{2-c_{0} \tau}, \quad c_{0}=\frac{4}{3}
$$

in the last relation. Therefore, dropping some positive terms in (3.33), we have

$$
\frac{d}{d t} \Psi(t) \leq C\|\theta\|_{L^{q, \infty}\left(\mathbb{R}^{3}\right)}^{p(1-\delta)} \Psi(t)^{4(1+2 \delta)} .
$$

In view of $\kappa \in[0,1]$, we know that $\delta \in[0,1]$. Finally, the proof is now based on applications of Lemma 2.1 and (1.10).

\subsection{Continuation theorem in anisotropic Lebesgue spaces}

Proof of (1) in Theorem 1.3. The interpolation inequality (2.9) in anisotropic Lebesgue spaces entails that

$$
\begin{aligned}
& \|\rho E\| \underset{L_{1}^{\frac{2 q_{1}}{q_{1}-2}} L_{2}^{\frac{2 q_{2}}{q_{2}-2}} L_{3}^{\frac{2 q_{3}}{q_{3}-2}}\left(\mathbb{R}^{3}\right)}{ } \leq C\|\rho E\|_{L^{2}\left(\mathbb{R}^{3}\right)}^{1-\left(\frac{1}{q_{2}}+\frac{1}{q_{2}}+\frac{1}{q_{3}}\right)}\|\rho E\|_{L^{\frac{m}{m_{1}}}\left(\mathbb{R}^{3}\right)}^{\left(\frac{1}{q_{1}}+\frac{1}{q_{3}}+\frac{1}{q_{3}}\right)} \\
& \leq C\|\rho E\|_{L^{2}\left(\mathbb{R}^{3}\right)}^{1-\left(\frac{1}{q_{1}}+\frac{1}{q_{2}}+\frac{1}{q_{3}}\right)}\|E\|_{L^{\frac{\vec{m}}{q_{1}}\left(\mathbb{R}^{3}\right)}}^{\left(\frac{1}{q_{2}}+\frac{1}{q_{3}}\right)},
\end{aligned}
$$

with $\vec{m}$ defined by $\vec{m}=\left(\frac{\frac{1}{q_{1}}+\frac{1}{q_{2}}+\frac{1}{q_{3}}}{\frac{1}{2}\left(\frac{1}{q_{1}}+\frac{1}{q_{2}}+\frac{1}{q_{3}}\right)-\frac{1}{q_{1}}}, \frac{\frac{1}{q_{1}}+\frac{1}{q_{2}}+\frac{1}{q_{3}}}{\frac{1}{2}\left(\frac{1}{q_{1}}+\frac{1}{q_{2}}+\frac{1}{q_{3}}\right)-\frac{1}{q_{2}}}, \frac{\frac{1}{q_{1}}+\frac{1}{q_{2}}+\frac{1}{q_{3}}}{\left.\frac{1}{q_{1}}+\frac{1}{q_{2}}+\frac{1}{q_{3}}\right)-\frac{1}{q_{3}}}\right)$.

The Sobolev embedding theorem (2.11) in anisotropic Lebesgue spaces guarantees

$$
\|\rho E\|_{L_{1}^{\frac{2 q_{1}}{q_{1}-2}} L_{2}^{\frac{2 q_{2}}{q_{2}-2}} L_{3}^{\frac{2 q_{3}}{q_{3}-2}}\left(\mathbb{R}^{3}\right)} \leq C\|\rho E\|_{L^{2}\left(\mathbb{R}^{3}\right)}^{1-\left(\frac{1}{q_{1}}+\frac{1}{q_{2}}+\frac{1}{q_{3}}\right)}\|\nabla E\|_{L^{2}\left(\mathbb{R}^{3}\right)}^{\left(\frac{1}{q_{1}}+\frac{1}{q_{2}}+\frac{1}{q_{3}}\right)}
$$

It follows from Hölder inequality (2.8) in anisotropic Lebesgue spaces that

$$
\begin{aligned}
\int \rho|u|^{2}|E|^{2} d x & \leq C\left\||u|^{2}\right\|_{L^{\frac{\vec{q}}{2}}\left(\mathbb{R}^{3}\right)}\left\|\rho E^{2}\right\|_{L_{1}^{\frac{q_{1}}{q_{1}-2}} L_{2}^{\frac{q_{2}}{q_{2}-2}} L_{3}^{\frac{q_{3}}{q_{3}-2}}\left(\mathbb{R}^{3}\right)} \\
& \leq C\|u\|_{L^{\vec{q}}\left(\mathbb{R}^{3}\right)}^{2}\|\rho E\|^{2}{ }_{L_{1}^{\frac{2 q_{1}}{q_{1}-2}} L_{2}^{\frac{2 q_{2}}{q_{2}-2}} L_{3}^{\frac{2 q_{3}}{q_{3}-2}}\left(\mathbb{R}^{3}\right)}^{2\left(\frac{1}{q_{1}}+\frac{1}{q_{2}}+\frac{1}{q_{3}}\right)}\|\rho E\|_{L^{2}\left(\mathbb{R}^{3}\right)}^{2-2\left(\frac{1}{q_{1}}+\frac{1}{q_{2}}+\frac{1}{q_{3}}\right)} \\
& \leq C\|u\|_{L^{\vec{q}}\left(\mathbb{R}^{3}\right)}^{2}\|\nabla E\|_{L^{2}\left(\mathbb{R}^{3}\right)} \\
& \leq C\|u\|_{L^{\vec{q}}\left(\mathbb{R}^{3}\right)}^{\left.\frac{2}{q_{1}}+\frac{1}{q_{2}}+\frac{1}{q_{3}}\right)}\|\rho E\|_{L^{2}\left(\mathbb{R}^{3}\right)}^{2}+\frac{C_{2}}{8}\|\nabla E\|_{L^{2}\left(\mathbb{R}^{3}\right)}^{2}
\end{aligned}
$$


By following exactly the lines of reasoning which led to (3.34), we infer that

$$
\|\nabla u\|_{L_{1}^{\frac{2 q_{1}}{q_{1}-2}} L_{2}^{\frac{2 q_{2}}{q^{2}-2}} L_{3}^{\frac{2 q_{3}}{q_{3}-2}}\left(\mathbb{R}^{3}\right)} \leq C\|\nabla u\|_{L^{2}\left(\mathbb{R}^{3}\right)}^{1-\left(\frac{1}{q_{1}}+\frac{1}{q_{2}}+\frac{1}{q_{3}}\right)}\|\nabla u\|_{L^{\frac{\vec{m}}{q_{1}}}\left(\mathbb{R}^{3}\right)}^{\left(\frac{1}{q_{2}}+\frac{1}{q_{3}}\right)}
$$

Taking advantage of (2.10), we conclude that

$$
\begin{aligned}
\|\nabla u\|_{L^{\vec{m}}\left(\mathbb{R}^{3}\right)} & \leq C\left(\|\operatorname{div} u\|_{L^{\vec{m}}\left(\mathbb{R}^{3}\right)}+\|\operatorname{curl} u\|_{L^{\vec{m}}\left(\mathbb{R}^{3}\right)}\right) \\
& \leq C\left(\|G\|_{L^{\vec{m}}\left(\mathbb{R}^{3}\right)}+\|P\|_{L^{\vec{m}}\left(\mathbb{R}^{3}\right)}+\|\operatorname{curl} u\|_{L^{\vec{m}}\left(\mathbb{R}^{3}\right)}\right) \\
& \leq C\left(\|G\|_{L^{\vec{m}}\left(\mathbb{R}^{3}\right)}+\|E\|_{L^{\vec{m}}\left(\mathbb{R}^{3}\right)}+\|\operatorname{curl} u\|_{L^{\vec{m}}\left(\mathbb{R}^{3}\right)}\right) \\
& \leq C\left(\|\nabla G\|_{L^{2}\left(\mathbb{R}^{3}\right)}+\|\nabla E\|_{L^{2}\left(\mathbb{R}^{3}\right)}+\|\nabla \operatorname{curl} u\|_{L^{2}\left(\mathbb{R}^{3}\right)}\right) \\
& \leq C\left(\|\rho \dot{u}\|_{L^{2}\left(\mathbb{R}^{3}\right)}+\|\nabla E\|_{L^{2}\left(\mathbb{R}^{3}\right)}\right),
\end{aligned}
$$

where the Sobolev embedding theorem (2.11) in anisotropic Lebesgue spaces was used again. This means that

$$
\|\nabla u\|{ }_{L_{1}^{\frac{2 q_{1}}{q_{1}-2}} L_{2}^{\frac{2 q_{2}}{q_{2}-2}} L_{3}^{\frac{2 q_{3}}{q_{3}-2}}\left(\mathbb{R}^{3}\right)} \leq C\|\nabla u\|_{L^{2}\left(\mathbb{R}^{3}\right)}^{1-\left(\frac{1}{q_{1}}+\frac{1}{q_{2}}+\frac{1}{q_{3}}\right)}\left(\|\rho \dot{u}\|_{L^{2}\left(\mathbb{R}^{3}\right)}+\|\nabla E\|_{L^{2}\left(\mathbb{R}^{3}\right)}\right)^{\left(\frac{1}{q_{1}}+\frac{1}{q_{2}}+\frac{1}{q_{3}}\right)} .
$$

The Hölder inequality in anisotropic Lebesgue spaces and the latter relation yield

$$
\begin{aligned}
\int \rho|u|^{2}|\nabla u|^{2} d x & \leq C\left\|\rho|u|^{2}\right\|_{L^{\frac{\vec{q}}{2}}\left(\mathbb{R}^{3}\right)}\left\||\nabla u|^{2}\right\|_{L_{1}^{\frac{q_{1}}{q_{1}-2}} L_{2}^{\frac{q_{2}}{q_{2}-2}} L_{3}^{\frac{q_{3}}{q_{3}-2}}\left(\mathbb{R}^{3}\right)} \\
& \leq C\left\||u|^{2}\right\|_{L^{\frac{\vec{q}}{2}}\left(\mathbb{R}^{3}\right)}\|\nabla u\|^{2} L_{1}^{\frac{2 q_{1}}{q_{1}-2}} L_{2}^{\frac{2 q_{2}}{q_{2}-2}} L_{3}^{\frac{2 q_{3}}{q_{3}-2}}\left(\mathbb{R}^{3}\right) \\
& \leq C\|u\|_{L^{\vec{q}}\left(\mathbb{R}^{3}\right)}^{2}\|\nabla u\|_{L^{2}\left(\mathbb{R}^{3}\right)}^{2-2\left(\frac{1}{q_{1}}+\frac{1}{q_{2}}+\frac{1}{q_{3}}\right)}\left(\|\dot{u}\|_{L^{2}\left(\mathbb{R}^{3}\right)}+\|\nabla E\|_{L^{2}\left(\mathbb{R}^{3}\right)}\right)^{2\left(\frac{1}{q_{1}}+\frac{1}{q_{2}}+\frac{1}{q_{3}}\right)} \\
& \leq C\|u\|_{L^{\vec{q}}\left(\mathbb{R}^{3}\right)}^{\frac{2}{1-\left(\frac{1}{q_{2}}+\frac{1}{q_{3}}\right)}}\|\nabla u\|_{L^{2}\left(\mathbb{R}^{3}\right)}^{2}+\frac{C_{2}}{8}\|\nabla E\|_{L^{2}\left(\mathbb{R}^{3}\right)}^{2}+\frac{1}{4}\|\rho \dot{u}\|_{L^{2}\left(\mathbb{R}^{3}\right)}^{2} .
\end{aligned}
$$

Inserting (3.36) and (3.39) into (3.18), we observe that

$$
\begin{aligned}
& \frac{d}{d t} \Phi(t)+\frac{1}{4} \int \rho|\dot{u}|^{2} d x+\frac{C_{2}}{4} \int|\nabla E|^{2} d x \\
\leq & C\|u\|_{L^{\vec{q}\left(\mathbb{R}^{3}\right)}}^{\frac{2}{1-\left(\frac{1}{q_{1}}+\frac{1}{q_{2}}+\frac{1}{q_{3}}\right)}}\left(\|\rho E\|_{L^{2}\left(\mathbb{R}^{3}\right)}^{2}+\|\nabla u\|_{L^{2}\left(\mathbb{R}^{3}\right)}^{2}\right) .
\end{aligned}
$$

To finish the proof, we now merely have to combine the Gronwall's inequality with (3.40) and (1.10).

Proof of (2) in Theorem 1.3. Estimating the last term of (3.26) by the Hölder inequality in anisotropic Lebesgue spaces, we see that

$$
\int \rho|u|^{2}|\theta|^{2} d x \leq C\|\theta\|_{L^{\vec{q}}\left(\mathbb{R}^{3}\right)}\|\sqrt{\rho} \theta\|_{L_{1}^{\frac{2 q_{1}}{q_{1}-1}} L_{2}^{\frac{2 q_{2}}{q_{2}-1}} L_{3}^{\frac{2 q_{3}}{q^{-1}}}\left(\mathbb{R}^{3}\right)}^{\left\|\sqrt{\rho}|u|^{2}\right\| L_{L_{1}^{\frac{2 q_{1}}{q_{1}-1}}} L_{2}^{\frac{2 q_{2}}{q_{2}-1}} L_{3}^{\frac{2 q_{3}}{q_{3}-1}}\left(\mathbb{R}^{3}\right)} .
$$


Due to the interpolation inequality (2.9), we get

$$
\begin{aligned}
\|\sqrt{\rho} \theta\| \|_{L_{1}^{\frac{2 q_{1}}{q_{1}-1}} \frac{2 q_{2}}{q_{2}-1}} \frac{2 q_{3}}{q_{3}^{3-1}}\left(\mathbb{R}^{3}\right) & \leq C\|\sqrt{\rho} \theta\|_{L^{2}\left(\mathbb{R}^{3}\right)}^{\left.1-\frac{1}{q_{1}}+\frac{1}{q_{2}}+\frac{1}{q_{3}}\right)}\|\sqrt{\rho} \theta\|_{L^{\frac{m}{q_{1}}\left(\mathbb{R}^{3}\right)}}^{\frac{1}{2}\left(\frac{1}{q_{2}}+\frac{1}{q_{3}}\right)} \\
& \leq C\|\sqrt{\rho} \theta\|_{L^{2}\left(\mathbb{R}^{3}\right)}^{1-\frac{1}{q_{1}}\left(\frac{1}{q_{1}}+\frac{1}{q_{2}}+\frac{1}{q_{3}}\right)}\|\nabla \theta\|_{L^{2}\left(\mathbb{R}^{3}\right)}^{\frac{1}{2}\left(\frac{1}{q_{1}}+\frac{1}{q_{2}}+\frac{1}{q_{3}}\right)} .
\end{aligned}
$$

Likewise,

$$
\left\|\sqrt{\rho}|u|^{2}\right\|_{L_{1}^{\frac{2 q_{1}}{q_{1}-1}} L_{2}^{\frac{2 q_{2}}{q_{2}-1}} L_{3}^{\frac{2 q_{3}}{q_{3}-1}}\left(\mathbb{R}^{3}\right)} \leq C\left\|\sqrt{\rho}|u|^{2}\right\|_{L^{2}\left(\mathbb{R}^{3}\right)}^{1-\frac{1}{2}\left(\frac{1}{q_{1}}+\frac{1}{q_{2}}+\frac{1}{q_{3}}\right)}\left\|\nabla|u|^{2}\right\|_{L^{2}\left(\mathbb{R}^{3}\right)}^{\frac{1}{2}\left(\frac{1}{q_{1}}+\frac{1}{q_{2}}+\frac{1}{q_{3}}\right)} .
$$

It is easy to see from the Young inequality that

$$
\begin{aligned}
& \int \rho|u|^{2}|\theta|^{2} d x \\
\leq & C\|\theta\|_{L^{\vec{q}}\left(\mathbb{R}^{3}\right)}\|\sqrt{\rho} \theta\|_{L^{2}\left(\mathbb{R}^{3}\right)}^{\left.1-\frac{1}{q_{1}}+\frac{1}{q_{2}}+\frac{1}{q_{3}}\right)}\left\|\sqrt{\rho}|u|^{2}\right\|_{L^{2}\left(\mathbb{R}^{3}\right)}^{\left.1-\frac{1}{q_{1}}+\frac{1}{q_{2}}+\frac{1}{q_{3}}\right)}\left(\|\nabla \theta\|_{L^{2}\left(\mathbb{R}^{3}\right)}^{\left(\frac{1}{q_{2}}+\frac{1}{q_{3}}\right)}+\left\|\nabla|u|^{2}\right\|_{L^{2}\left(\mathbb{R}^{3}\right)}^{\left(\frac{1}{q_{2}}+\frac{1}{q_{3}}\right)}\right) \\
\leq & C\|\theta\|_{L^{\vec{q}\left(\mathbb{R}^{3}\right)}}^{\frac{\left.2-\frac{1}{q_{2}}+\frac{1}{q_{3}}\right)}{2-\left(\frac{1}{2}\right)}}\left(\|\sqrt{\rho} \theta\|_{L^{2}\left(\mathbb{R}^{3}\right)}^{2}+\left\|\sqrt{\rho}|u|^{2}\right\|_{L^{2}\left(\mathbb{R}^{3}\right)}^{2}\right)+\frac{1}{16}\left(\|\nabla \theta\|_{L^{2}\left(\mathbb{R}^{3}\right)}^{2}+\left\|\nabla|u|^{2}\right\|_{L^{2}\left(\mathbb{R}^{3}\right)}^{2}\right) .
\end{aligned}
$$

Similarly, we conclude that

$$
\begin{aligned}
& \int \rho|\theta|^{3} d x \leq C\|\theta\|_{L^{\vec{q}}\left(\mathbb{R}^{3}\right)}\|\sqrt{\rho} \theta\|^{2} \frac{2 q_{1}}{L_{1}^{q_{1}-1}} L_{2}^{\frac{2 q_{2}}{q_{2}-1}} L_{3}^{\frac{2 q_{3}}{q_{3}-1}}\left(\mathbb{R}^{3}\right) \\
& \leq C\|\theta\|_{L^{\vec{q}\left(\mathbb{R}^{3}\right)}}\|\sqrt{\rho} \theta\|_{L^{2}\left(\mathbb{R}^{3}\right)}^{2-\left(\frac{1}{q_{1}}+\frac{1}{q_{2}}+\frac{1}{q_{3}}\right)}\|\nabla \theta\|_{L^{2}\left(\mathbb{R}^{3}\right)}^{\left(\frac{1}{q_{1}}+\frac{1}{q_{2}}+\frac{1}{q_{3}}\right)} \\
& \leq C\|\theta\|_{L^{\left(\vec{q}\left(\mathbb{R}^{3}\right)\right.}}^{\frac{\left.\frac{1}{q_{2}}+\frac{1}{q_{2}}+\frac{1}{q_{3}}\right)}{2 .}}\|\sqrt{\rho} \theta\|_{L^{2}\left(\mathbb{R}^{3}\right)}^{2}+\frac{1}{16}\|\nabla \theta\|_{L^{2}\left(\mathbb{R}^{3}\right)}^{2} .
\end{aligned}
$$

As a consequence of the above estimates and (3.26), we see that

$$
\begin{aligned}
\frac{d}{d t} \Psi(t)+\frac{\kappa}{4} \int|\nabla \theta|^{2} d x+ & \frac{1}{2} \int \rho|\dot{u}|^{2} d x+\frac{1}{4} \int_{\mathbb{R}^{3} \cap\{|u|>0\}}|u|^{2}|\nabla u|^{2} \\
& \leq C\|\theta\|_{L^{\frac{\vec{q}}{\left(\mathbb{R}^{3}\right)}}}^{\frac{2}{\left.q_{1}+\frac{1}{q_{2}}+\frac{1}{q_{3}}\right)}}\left(\|\sqrt{\rho} \theta\|_{L^{2}\left(\mathbb{R}^{3}\right)}^{2}+\left\|\sqrt{\rho}|u|^{2}\right\|_{L^{2}\left(\mathbb{R}^{3}\right)}^{2}\right) .
\end{aligned}
$$

Combining this and Gronwall's lemma, we are now able to complete the proof.

\section{Nonhomogeneous incompressible Navier-Stokes equations}

We proceed with the nonhomogeneous incompressible Navier-Stokes equations similarly as in the previous proof.

Proof of Theorem 1.4. Taking the $L^{2}$ inner product of the system with $u_{t}$ and integrating by parts, we have

$$
\frac{\mu}{2} \frac{d}{d t} \int|\nabla u|^{2} d x+\int \rho\left|u_{t}\right|^{2} d x=-\int \rho u \cdot \nabla u \cdot u_{t} d x
$$


From the Young inequality, we infer that

$$
-\int \rho u \cdot \nabla u \cdot u_{t} d x \leq \frac{1}{4} \int \rho\left|u_{t}\right|^{2} d x+C \int \rho|u|^{2}|\nabla u|^{2} d x .
$$

Next, we write (1.20) in the form

$$
-\mu \Delta u+\nabla \Pi=-\rho u_{t}-\rho u \cdot \nabla u .
$$

Resorting to the classical Stokes estimate, we discover that

$$
\left\|\nabla^{2} u\right\|_{L^{2}\left(\mathbb{R}^{3}\right)}^{2} \leq C_{6}\left(\left\|\rho u_{t}\right\|_{L^{2}\left(\mathbb{R}^{3}\right)}^{2}+\|\rho u \cdot \nabla u\|_{L^{2}\left(\mathbb{R}^{3}\right)}^{2}\right),
$$

namely,

$$
\frac{1}{4 C_{6}}\left\|\nabla^{2} u\right\|_{L^{2}\left(\mathbb{R}^{3}\right)}^{2} \leq \frac{1}{4}\left(\left\|\rho u_{t}\right\|_{L^{2}\left(\mathbb{R}^{3}\right)}^{2}+\|\rho u \cdot \nabla u\|_{L^{2}\left(\mathbb{R}^{3}\right)}^{2}\right) .
$$

This in turn implies

$$
\frac{1}{2} \int \rho\left|u_{t}\right|^{2} d x+\frac{\mu}{2} \frac{d}{d t} \int|\nabla u|^{2} d x+\frac{1}{4 C_{6}} \int\left|\nabla^{2} u\right|^{2} d x \leq C \int \rho|u|^{2}|\nabla u|^{2} d x .
$$

(1) Repeating the process of deduction in (3.21), we obtain

$$
\begin{aligned}
\int \rho|u|^{2}|\nabla u|^{2} d x & \leq\left\||u|^{2}\right\|_{L^{\frac{q}{2}, \infty}\left(\mathbb{R}^{3}\right)}\left\||\nabla u|^{2}\right\|_{L^{\frac{q}{q-2}, 1}\left(\mathbb{R}^{3}\right)} \\
& \leq\|u\|_{L^{q, \infty}\left(\mathbb{R}^{3}\right)}^{2}\|\nabla u\|_{L^{\frac{2 q}{q-2}, 2}\left(\mathbb{R}^{3}\right)}^{2} \\
& \leq C\|u\|_{L^{q, \infty}\left(\mathbb{R}^{3}\right)}^{2}\left\|\nabla^{2} u\right\|_{L^{2}\left(\mathbb{R}^{3}\right)}^{\frac{6}{q}}\|\nabla u\|_{L^{2}\left(\mathbb{R}^{3}\right)}^{2-\frac{6}{q}} \\
& \leq C\|u\|_{L^{q, \infty}\left(\mathbb{R}^{3}\right)}^{\frac{2 q}{q-3}}\|\nabla u\|_{L^{2}\left(\mathbb{R}^{3}\right)}^{2}+\frac{1}{32}\left\|\nabla^{2} u\right\|_{L^{2}\left(\mathbb{R}^{3}\right)}^{2} .
\end{aligned}
$$

Lemma 2.2 with $a=1, b=6$ and $c_{0}=4$ gives

$$
\begin{aligned}
\|u\|_{L^{q_{\tau}, \infty}\left(\mathbb{R}^{3}\right)}^{p_{\tau}} & \leq\|u\|_{L^{q, \infty}\left(\mathbb{R}^{3}\right)}^{p(1-\tau)}\|u\|_{L^{6, \infty}\left(\mathbb{R}^{3}\right)}^{4 \tau} \\
& \leq C\|u\|_{L^{q, \infty}\left(\mathbb{R}^{3}\right)}^{p(1-\tau)}\|u\|_{L^{6,2}\left(\mathbb{R}^{3}\right)}^{4 \tau} \\
& \leq C\|u\|_{L^{q, \infty}\left(\mathbb{R}^{3}\right)}^{p(1-\tau)}\|\nabla u\|_{L^{2}\left(\mathbb{R}^{3}\right)}^{4 \tau} .
\end{aligned}
$$

Plugging these estimates into (4.1), we find

$$
\frac{1}{2} \int \rho\left|u_{t}\right|^{2} d x+\frac{\mu}{2} \frac{d}{d t} \int|\nabla u|^{2} d x+\frac{1}{8 C_{6}} \int\left|\nabla^{2} u\right|^{2} d x \leq C\|u\|_{L^{q, \infty}\left(\mathbb{R}^{3}\right)}^{p(1-\tau)}\|\nabla u\|_{L^{2}\left(\mathbb{R}^{3}\right)}^{4 \tau+2} .
$$

Applying Lemma 2.1 to (4.2) and using (1.21), we complete the proof.

(2) Exactly as in the derivation of (3.36), we have

$$
\begin{aligned}
& \int \rho|u|^{2}|\nabla u|^{2} d x \leq C\left\||u|^{2}\right\|_{L^{\frac{\vec{q}}{2}}\left(\mathbb{R}^{3}\right)}\left\||\nabla u|^{2}\right\|_{L_{1}^{\frac{q_{1}}{q_{1}-2}} L_{2}^{\frac{q_{2}}{q_{2}-2}} L_{3}^{\frac{q_{3}}{q_{3}-2}}\left(\mathbb{R}^{3}\right)} \\
& \leq C\|u\|_{L^{\vec{q}}\left(\mathbb{R}^{3}\right)}^{2}\|\nabla u\|^{2} \frac{2 q_{1}}{L_{1}^{q_{1}-2}} L_{2}^{\frac{2 q_{2}}{q_{2}-2}} L_{3}^{\frac{2 q_{3}}{q_{3}-2}}\left(\mathbb{R}^{3}\right) \\
& \leq C\|u\|_{L^{\vec{q}}\left(\mathbb{R}^{3}\right)}^{2}\left\|\nabla^{2} u\right\|_{L^{2}\left(\mathbb{R}^{3}\right)}^{2\left(\frac{1}{q_{2}}+\frac{1}{q_{3}}+\frac{1}{q_{3}}\right)}\|\nabla u\|_{L^{2}\left(\mathbb{R}^{3}\right)}^{2-2\left(\frac{1}{q_{1}}+\frac{1}{q_{2}}+\frac{1}{q_{3}}\right)} \\
& \leq C\|u\|_{L^{\left(\vec{q}\left(\mathbb{R}^{3}\right)\right.}}^{\frac{2}{1-\left(\frac{1}{q_{1}}+\frac{1}{q_{2}}+\frac{1}{q_{3}}\right)}}\|\nabla u\|_{L^{2}\left(\mathbb{R}^{3}\right)}^{2}+\frac{1}{8 C_{6}}\left\|\nabla^{2} u\right\|_{L^{2}\left(\mathbb{R}^{3}\right)}^{2} .
\end{aligned}
$$


As a consequence, we get

$$
\frac{1}{2} \int \rho\left|u_{t}\right|^{2} d x+\frac{\mu}{2} \frac{d}{d t} \int|\nabla u|^{2} d x+\frac{1}{8 C_{6}} \int\left|\nabla^{2} u\right|^{2} d x \leq C\|u\|_{L^{(\vec{q}}\left(\mathbb{R}^{3}\right)}^{\frac{1}{1-\left(\frac{1}{q_{2}}+\frac{1}{q_{3}}+\frac{1}{q_{3}}\right)}}\|\nabla u\|_{L^{2}\left(\mathbb{R}^{3}\right)}^{2} .
$$

(3) In view of the Hölder inequality and Sobolev inequality, we get

$$
\begin{aligned}
\int \rho|u|^{2}|\nabla u|^{2} d x & \leq\left\|\left.u\right|^{2}\right\|_{L^{3}\left(\mathbb{R}^{3}\right)}\left\||\nabla u|^{2}\right\|_{L^{\frac{3}{2}}\left(\mathbb{R}^{3}\right)} \\
& \leq\|u\|_{L^{6}\left(\mathbb{R}^{3}\right)}^{2}\|\nabla u\|_{L^{3}\left(\mathbb{R}^{3}\right)}^{2} \\
& \leq C\|\nabla u\|_{L^{3}\left(\mathbb{R}^{3}\right)}^{2}\|\nabla u\|_{L^{2}\left(\mathbb{R}^{3}\right)}^{2}
\end{aligned}
$$

This leads to

$$
\frac{1}{2} \int \rho\left|u_{t}\right|^{2} d x+\frac{\mu}{2} \frac{d}{d t} \int|\nabla u|^{2} d x+\frac{1}{8 C_{6}} \int\left|\nabla^{2} u\right|^{2} d x \leq C\|\nabla u\|_{L^{3}\left(\mathbb{R}^{3}\right)}^{2}\|\nabla u\|_{L^{2}\left(\mathbb{R}^{3}\right)}^{2} .
$$

With (4.1), (4.3) and (4.4) in hand, employing classical Gronwall's lemma, we complete the proof by (1.21).

\section{Acknowledgement}

Wang was partially supported by the National Natural Science Foundation of China under grant (No. 11971446 and No. 11601492). Wei was partially supported by the National Natural Science Foundation of China under grant (No. 11601423, No. 11701450, No. 11701451, No. 11771352, No. 11871057) and Scientific Research Program Funded by Shaanxi Provincial Education Department (Program No. 18JK0763). The research of Wu was partially supported by the National Natural Science Foundation of China under grant No. 11771423 and No. 11671378. Ye is supported by the NSFC (No. 11701145).

\section{References}

[1] A. Benedek and R. Panzone. The space $L^{p}$ with mixed norm. Duke Math. J. 28 (1961) $301-324$.

[2] J. Bergh and J. Löfström, Interpolation Spaces. Springer-Verlag, Berlin, 1976.

[3] S. Bosia, V. Pata and J. Robinson, A Weak- $L^{p}$ Prodi-Serrin Type Regularity Criterion for the Navier-Stokes Equations. J. Math. Fluid Mech., 16 (2014), 721-725.

[4] J. A. Carrillo and L. C F. Ferreira, Self-similar solutions and large time asymptotics for the dissipative quasi-geostrophic equation. Monatsh. Math., 151 (2007) 111-142.

[5] Z. Chen and W. G. Price, Blow-up rate estimates for weak solutions of the NavierStokes equations. (English summary) R. Soc. Lond. Proc. Ser. A Math. Phys. Eng. Sci. 457 (2001), 2625-2642. 
[6] Y. Cho, H. J. Choe and H. Kim, Unique solvability of the initial boundary value problems for compressible viscous fluid, J. Math. Pures Appl., 83 (2004), 243-275.

[7] Y. Cho and H. Kim, Existence results for viscous polytropic fluids with vacuum. J. Differential Equations., 228 (2006), 377-411.

[8] H. J. Choe and H. Kim, Strong solutions of the Navier-Stokes equations for nonhomogeneous incompressible fluids, Comm. PDE. 28 (2003) 1183-1201.

[9] H. Choe and M. Yang, Blow up criteria for the compressible Navier-Stokes equations. Mathematical analysis in fluid mechanics-selected recent results, 65-84, Contemp. Math., 710, Amer. Math. Soc., Providence, RI, 2018.

[10] E. Feireisl, Dynamics of Viscous Compressible Fluids, Oxford Univ. Press, Oxford, (2004).

[11] Z. Guo, M. Caggio and Z. Skalàk, Regularity criteria for the Navier-Stokes equations based on one component of velocity. Nonlinear Anal. Real World Appl. 35 (2017), 379-396.

[12] S. Gustafson, K. Kang and T. Tsai, Interior regularity criteria for suitable weak solutions of the Navier-Stokes equations, Commun. Math. Phys. 273 (2007), 161-176.

[13] L. Grafakos, Classical Fourier analysis. 2nd Edition, Springer, 2008.

[14] X. Huang and J. Li, Serrin-type blowup criterion for viscous, compressible, and heat conducting Navier-Stokes and magnetohydrodynamic flows. Comm. Math. Phys. 324 (2013), 147-171.

[15] X. Huang, J. Li and Y. Wang, Serrin-type blowup criterion for full compressible NavierStokes system. Arch. Ration. Mech. Anal. 207 (2013), 303-316

[16] X. Huang, J. Li and Z. Xin, Serrin type criterion for the three-dimensional compressible flows. SIAM J. Math. Anal. 43 (2011), 1872-1886.

[17] X. Huang and Z. Xin, A blow-up criterion for classical solutions to the compressible Navier-Stokes equations. Sci. China Math., 53 (2010), 671-686.

[18] Q. Jiu, Y. Wang and Y. Ye, Refined blow up criteria for the full compressible NavierStokes equations involving temperature, arXiv: 1906.07478

[19] X. Ji, Y. Wang and W. Wei, New regularity criteria based on pressure or gradient of velocity in Lorentz spaces for the 3D Navier-Stokes equations. J. Math. Fluid Mech., accepted for publication. Also posted in arXiv:1909.09960

[20] H. Kim and H. Kozono, Interior regularity criteria in weak spaces for the Navier-Stokes equations. Manuscripta Math., 115 (2004), 85-100.

[21] H. Kim, A blow-up criterion for the nonhomogeneous incompressible Navier-Stokes equations, SIAM J. Math. Anal. 37 (2006) 1417-1434.

[22] Z. Lei and Z. Xin, On scaling invariance and type-I singularities for the compressible Navier-Stokes equations. Sci. China Math., 2019, 1-16. 
[23] P. L. Lions, Mathematical topics in fluid mechanics. Vol. 2. Compressible models. Oxford University Press, New York, (1998).

[24] J. Malý, Advanced theory of differentiation-Lorentz spaces, March 2003 http://www.karlin.mff.cuni.cz/〜 maly/lorentz.pdf.

[25] R. O'Neil, Convolution operaters and $L^{p, q}$ spaces. Duke Math J., 30 (1963), 129-142.

[26] J.L. Rubio de Francia, F.J. Ruiz and J.L. Torrea, Calderon-Zygmund theory for operator-valued kernels, Adv. Math. 62 (1986), 7-48.

[27] J. Serrin, On the interior regularity of weak solutions of the Navier-Stokes equations, Arch. Rational Mech. Anal. 9 (1962), 187-195.

[28] Y. Sun, C. Wang and Z. Zhang, A Beale-Kato-Majda blow-up criterion for the 3D compressible Navier-Stokes equations. J. Math. Pures Appl. 95 (2011), 36-47.

[29] Y. Sun, C. Wang and Z. Zhang, A Beale-Kato-Majda criterion for three dimensional compressible viscous heat-conductive flows. Arch. Ration. Mech. Anal. 201 (2011), $727-742$.

[30] Y. Sun and Z. Zhang, Blow-up criteria of strong solutions and conditional regularity of weak solutions for the compressible Navier-Stokes equations. Handbook of mathematical analysis in mechanics of viscous fluids, 2263-2324, Springer, Cham, 2018.

[31] M. Struwe, On partial regularity results for the Navier-Stokes equations, Comm. Pure Appl. Math., 41 (1988), 437-458.

[32] H. Sohr, Aregularity class for the Navier-Stokes equations in Lorentz spaces. J. Evol. Equ., 1 (2001), 441-467.

[33] L. Tartar, Imbedding theorems of Sobolev spaces into Lorentz spaces. Bollettino dell'Unione Matematica Italiana, 1 (1998), 479-500.

[34] Y. Wang, G. Wu and D. Zhou, $\varepsilon$-regularity criteria in anisotropic Lebesgue spaces and Leray's self-similar solutions to the 3D Navier-Stokes equations. arXiv:1901.01510v3.

[35] Y. Wang, W. Wei and H. Yu, $\varepsilon$-regularity criteria in Lorentz spaces to the 3D NavierStokes equations. arXiv:1909.09957.

[36] Y. Wang, Weak Serrin-type blowup criterion for three-dimensional nonhomogeneous viscous incompressible heat conducting flows, Physica D. 2019.

[37] H. Wen and C. Zhu, Blow-up criterions of strong solutions to 3D compressible NavierStokes equations with vacuum, Adv. Math. 248 (2013), 534-572.

[38] X. Xu and J. Zhang. A blow-up criterion for 3D compressible magnetohydrodynamic equations with vacuum. Math. Models Method. Appl. Sci. 22 (2012) 1150010.

[39] X. Zheng, A regularity criterion for the tridimensional Navier-Stokes equations in term of one velocity component. J. Differential Equations, 256 (2014), 283-309. 